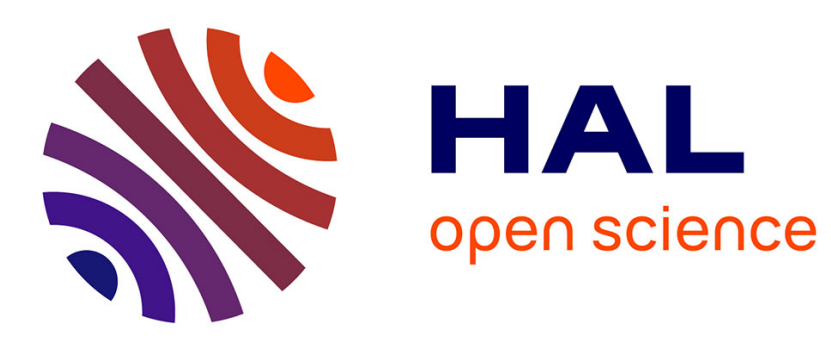

\title{
Thermal recovery from a fractured medium in local thermal non-equilibrium
}

\author{
Rachel Gelet, Benjamin Loret, Nasser Khalili
}

\section{To cite this version:}

Rachel Gelet, Benjamin Loret, Nasser Khalili. Thermal recovery from a fractured medium in local thermal non-equilibrium. International Journal for Numerical and Analytical Methods in Geomechanics, 2013, 37 (15), pp.2471-2501. 10.1002/nag.2145 . hal-00919449

\section{HAL Id: hal-00919449 \\ https://hal.science/hal-00919449}

Submitted on 16 Dec 2013

HAL is a multi-disciplinary open access archive for the deposit and dissemination of scientific research documents, whether they are published or not. The documents may come from teaching and research institutions in France or abroad, or from public or private research centers.
L'archive ouverte pluridisciplinaire $\mathbf{H A L}$, est destinée au dépôt et à la diffusion de documents scientifiques de niveau recherche, publiés ou non, émanant des établissements d'enseignement et de recherche français ou étrangers, des laboratoires publics ou privés. 


\title{
Thermal recovery from a fractured medium in local thermal non-equilibrium
}

\author{
Rachel Gelet ${ }^{\mathrm{a}, \mathrm{b}, *}$, Benjamin Loret $^{\mathrm{a}}$, Nasser Khalili $^{\mathrm{b}}$ \\ ${ }^{a}$ Laboratoire Sols, Solides, Structures, B.P. 53X, 38041 Grenoble Cedex, France \\ ${ }^{b}$ School of Civil and Environmental Engineering, The University of New South Wales, Sydney 2052, Australia
}

\begin{abstract}
Thermal recovery from a hot dry rock reservoir viewed as a deformable fractured medium is investigated with a focus on the assumption of local thermal non-equilibrium (LTNE). Hydraulic diffusion, thermal diffusion, forced convection and deformation are considered in a two-phase framework, the solid phase being made by impermeable solid blocks separated by saturated fractures. The finite element approximation of the constitutive and field equations is formulated and applied to obtain the response of a generic hot dry rock reservoir to circulation tests. A change of time profile of the outlet fluid temperature is observed as the fracture spacing increases, switching from a single-step pattern to a double-step pattern, a feature which is viewed as characteristic of established local thermal non-equilibrium. A dimensionless number is proposed to delineate between local thermal equilibrium (LTE) and non-equilibrium. This number embodies local physical properties of the mixture, elements of the geometry of the reservoir and the production flow rate. All the above properties being fixed, the resulting fracture spacing threshold between LTNE and LTE is found to decrease with increasing porosity or fluid velocity. The thermally induced effective stress is tensile near the injection well, illustrating the thermal contraction of the rock, while the pressure contribution of the fracture fluid is negligible during the late period.
\end{abstract}

Keywords: enhanced geothermal system, hot dry rock, coupled problems, thermal stress, local thermal non-equilibrium

\section{Introduction}

Geothermal extraction from deep hot dry rock (HDR) reservoirs may become a viable alternative to grey energies in the "far future" [1]. The production of geothermal energy is achieved by cold water injection, in fractured igneous rocks (originally with low matrix permeability), at sites where the vertical temperature gradient is favorable. Of crucial importance to the economic viability of these HDR reservoirs is the knowledge of thermal output evolution, induced thermal stress and fluid loss, at various time scales of the circulation tests [2].

Thermo-poro-elastic mechanisms in addition to chemical, damage and plastic processes can play a significant role on the overall behavior of the HDR reservoirs [3].

Closed form solutions for the prediction of uncoupled reservoir depletion have been presented by a number of investigators, where heat transfer is dominated by convection in the fluid phase and by conduction in the solid phase $[4,5,6]$. Ghassemi et al. [7] provided an uncoupled three-dimensional integral equation for calculating thermally induced stresses, highlighting the importance of induced thermal stress, without the burden of discretizing the reservoir. Notable contributions have also been made by Wang and Dusseault [8] on the effects of convective-conductive heat flow on the stresses near a cylindrical wellbore.

\footnotetext{
*Principal corresponding author

Email addresses: rachel.gelet@gmail.com (Rachel Gelet), benjamin.loret@hmg.inpg.fr (Benjamin Loret), n.khalili@unsw.edu.au (Nasser Khalili)
} 
Coupled thermo-hydro-mechanical analyses for geothermal systems are scarce $[9,10]$ and focus mainly on partially coupled systems in an effort to implement a precise fracture network through a system of discrete discontinuities [11, 12], to couple free and forced convection [13], or to characterize joint closure with a stress dependent law $[14,15]$ or stress-dissolution/precipitation effects [16].

Alternatively, comprehensive fully coupled thermo-hydro-mechanical formulations based on the mixture theory have been presented by Loret and Khalili [17] and Khalili and Loret [18] for unsaturated porous media, with emphasis on the importance of an appropriate definition of the effective stress. While thermo-chemomechanical couplings associated with mineral precipitation/dissolution may alter the fracture aperture [19], they have not been accounted for in this study which focuses on local thermal non-equilibrium.

A key factor influencing geothermal energy recovery is the difference in characteristic times between diffusion in the solid phase and convection in the fluid phase. The thermally induced effective stress which results from these two contributions may lead to permeability change and fluid loss. Thermal shrinkage and pressure drop, across the body of the reservoir, occur at various time scales and the understanding of their interaction requires the simulation of a continuum mixture in local thermal non-equilibrium [20, 21].

This paper is aimed at presenting a fully coupled finite element formulation for a thermo-elastic fractured medium in local thermal non-equilibrium. The fractured medium is described as a porous mixture composed of a solid phase and a fluid phase. The solid matrix is made of impermeable solid blocks surrounded by saturated fractures. Numerical solutions are obtained by enforcing the balances of mass, momentum and energy. A summary of the governing differential equations is provided in Sect. 2. The weak form of the governing equations, the discretization and time-integration procedures to solve the coupled equations through a finite element method are detailed in Sect. 3. The primary variables are the displacements, the pressure of the fluid, the temperature of the solid phase and the temperature of the fluid. The resulting system of equations is used to address a generic HDR reservoir subjected to temperature gradients and to various external heat supplies (Sect.4). An attempt to define a dimensionless threshold above which a LTNE analysis is required is exposed (Sect.5). The threshold embodies local physical properties of the mixture, elements of the geometry of the reservoir and the production flow rate. When interpreted with help of this threshold, the simulations of geothermal energy recovery highlight quite distinct time profiles of the outlet temperature, depending whether local thermal equilibrium (LTE) or local thermal non-equilibrium (LTNE) hold in the reservoir. A change of time profile of the outlet fluid temperature is observed as the fracture spacing increases, switching from a single-step pattern to a double-step pattern, a feature which is interpreted as a characteristic of established local thermal non-equilibrium. The calibrated model is used to obtain the thermal output and the thermally induced effective stress response to circulation tests, which are compared with data obtained at the Fenton Hill HDR reservoir (Sect. 6).

\section{The two temperature thermoporoelastic model}

Geothermal energy is produced by circulating a fluid through the fractured reservoir within a single porosity conceptual framework. This continuum treatment requires the mixture to be in thermal nonequilibrium so that the processes acting on the fluid phase and on the solid phase are properly accounted for.

\subsection{Model assumptions}

Local thermal non-equilibrium between the phases holds at all points of the simulated zone. Each phase $k$ is endowed with its own temperature $T_{k}$ and its own material properties. Local thermal nonequilibrium (LTNE) emanates from the contrast between the rapid convection of heat by the moving fluid in the fractures and the slow diffusion of heat through the solid matrix. Indeed, the characteristic time associated with convection is several orders of magnitude smaller than the characteristic times of diffusion in both solid skeleton and fluid, Sect.3.3.

In order to concentrate efforts on the heat exchange between phases, a number of restrictions are adopted in the development of the model: 
1. The material properties of each phase, namely, the porosities, the permeabilities, the densities, the viscosities, the conductivities, the specific heat capacities, the hydraulic compressibility, the coefficients of thermal expansion, as well as the solid-fluid specific heat transfer coefficient, are assumed to remain constant with time;

2. Density driven convection are neglected. In addition, the thermal boundary resistance (Kapitza resistance), the solid surface wettability and the lagging response [22] are considered negligible in the lifetime of the reservoir;

3. The additional stiffness induced by the initial fluid pressure is not accounted for either;

4. The flow regime remains laminar.

\subsection{Governing equations}

With the indices $s$ and $f$ referring to the solid and to the fluid respectively, the governing field equations of the two phase mixture in local thermal non-equilibrium enforce the balance of momentum of the mixture as a whole, the balance of mass for the fluid phase and the balances of energy of the solid and fluid phases $[23,24]$,

$$
\begin{aligned}
& \operatorname{div} \boldsymbol{\sigma}+\rho \mathbf{g}=\mathbf{0}, \\
& \operatorname{div} \mathbf{j}_{f}+\mathcal{J}_{f}=0, \quad \mathcal{J}_{f} \equiv n_{f} \frac{1}{\rho_{f}} \frac{\mathrm{d}^{f} \rho_{f}}{d t}+\frac{1}{V} \frac{\mathrm{d}^{s} V_{f}}{\mathrm{~d} t}, \\
& \operatorname{div} \mathbf{q}_{s}+\mathcal{Q}_{s}=0, \quad \mathcal{Q}_{s} \equiv T_{s} \frac{\mathrm{d}^{s} s^{s}}{\mathrm{~d} t}+\hat{e}_{s f}, \\
& \operatorname{div} \mathbf{q}_{f}+\mathcal{Q}_{f}=0, \quad \mathcal{Q}_{f} \equiv T_{f} \frac{\mathrm{d}^{s} s^{f}}{\mathrm{~d} t}-\hat{e}_{s f}+\rho_{f} \mathbf{j}_{f} \cdot \nabla H_{f},
\end{aligned}
$$

where $\boldsymbol{\sigma}$ is the total stress, $\mathbf{g}$ is the gravity, $\mathbf{j}_{f}$ is the apparent volume flux of the fluid relative to the solid skeleton, and $\mathbf{q}_{k}$ is the apparent heat flux through the phase $k$. The rate of energy transfer (or exchange) from the solid phase to the fluid phase $\hat{e}_{s f}$ is defined by the constitutive equation (13) below.

The field equations feature intrinsic quantities like the mass density $\rho_{k}$, the specific entropy $S_{k}[\mathrm{~J} / \mathrm{kg} \cdot \mathrm{K}]$ and the specific enthalpy $H_{k}[\mathrm{~J} / \mathrm{kg}]$ of phase $k$. At variance, with $n_{k}=V_{k} / V$ the volume fraction of phase $k$ in the mixture, $\rho^{k}=n_{k} \rho_{k}$ and $s^{k}=\rho^{k} S_{k}$ are apparent quantities that represent respectively the mass and entropy of the phase $k$ per unit current volume of mixture.

Finally, the displacement vector of the solid skeleton is denoted by $\mathbf{u}$, while $\mathbf{v}_{k}$ denotes the velocity vector of phase $k$, so that the apparent volume flux of the fluid relative to the solid skeleton $\mathbf{j}_{f}$ is equal to $n_{f}\left(\mathbf{v}_{f}-\mathbf{v}_{s}\right)$.

The initial configuration, which serves as a reference, represents a state in mechanical and thermal equilibrium in which stress, strain and fluid pressure may be non zero. The solid temperature and the fluid temperature are initially equal to $T^{0}$. Departure from this reference state is denoted by the symbol $\Delta(\cdot)$.

The thermo-elastic mixture remains isotropic in both elastic and thermal properties. The shear behavior is accounted for by the second Lamé's constant $\mu_{\mathrm{DS}}$ of the drained solid skeleton while the fluid does not react to shear stresses. The elastic relationship links the elastic strain $\boldsymbol{\epsilon}^{e l}$ to the effective stress $\overline{\boldsymbol{\sigma}}$,

$$
\operatorname{tr} \boldsymbol{\epsilon}^{e l}=c_{\mathrm{DS}} \frac{\operatorname{tr} \overline{\boldsymbol{\sigma}}}{3}, \quad \operatorname{dev} \boldsymbol{\epsilon}^{e l}=\frac{\operatorname{dev} \overline{\boldsymbol{\sigma}}}{2 \mu_{\mathrm{DS}}},
$$

where tr and dev denote respectively the trace and deviator operators. Actually, the total stress $\boldsymbol{\sigma}$ and the effective stress $\overline{\boldsymbol{\sigma}}$,

$$
\overline{\boldsymbol{\sigma}}=\boldsymbol{\sigma}+\xi_{f} p_{f} \mathbf{I}
$$

have identical deviatoric parts. Here $p_{f}$ is the fluid pressure, and $\xi_{f}=1-c_{s} / c_{\mathrm{DS}}$ is Biot's coefficient expressed in terms of the compressibilities $[1 / \mathrm{Pa}]$ of the solid skeleton $c_{\mathrm{DS}}$ and of the solid constituent $c_{s}$. The compressibility $c_{\mathrm{DS}}$ and the Lamé's constants $[\mathrm{Pa}]$ of the drained solid $\lambda_{\mathrm{DS}}$ and $\mu_{\mathrm{DS}}$ are associated with the drained Young's modulus $E$ and the drained Poisson's ratio $\nu$ through the standard relationships,

$$
c_{\mathrm{DS}}=\frac{3(1-2 \nu)}{E}, \quad \lambda_{\mathrm{DS}}=\frac{E \nu}{(1+\nu)(1-2 \nu)}, \quad \mu_{\mathrm{DS}}=\frac{E}{2(1+\nu)} .
$$


The total strain of the solid skeleton derives from the displacement vector $\mathbf{u}=\left(u_{i}\right)$, namely componentwise $\epsilon_{i j}=\frac{1}{2}\left(\partial u_{i} / \partial x_{j}+\partial u_{j} / \partial x_{i}\right)$. It is contributed additively by the elastic strain and by the thermal strain,

$$
\boldsymbol{\epsilon}=\boldsymbol{\epsilon}^{e l}+c_{T} \Delta T_{s} \mathbf{I}
$$

with $c_{T}[1 / \mathrm{K}]$ the volumetric thermal expansion coefficient of the solid skeleton.

Aside the deviatoric components which are governed by $(2)_{2}$, the mixed thermo-mechanical constitutive system relates the isotropic part of the total stress $\operatorname{tr} \boldsymbol{\sigma} / 3$, the volume content of the fluid $v^{f}=V_{f} / V^{0}$, and the apparent entropy of the solid $s^{s}$ to the volume change of the solid skeleton tr $\epsilon$, to the fluid pressure $p_{f}$ and to the solid temperature $T_{s}$ by $[25,26]$

$$
\begin{aligned}
-\frac{\operatorname{tr} \boldsymbol{\sigma}}{3} & =-\frac{1}{c_{\mathrm{DS}}} \operatorname{tr} \boldsymbol{\epsilon}+\xi_{f} p_{f}+\frac{c_{T}}{c_{\mathrm{DS}}} \Delta T_{s} \\
\Delta v^{f} & =\xi_{f} \operatorname{tr} \boldsymbol{\epsilon}+\left(\xi_{f}-n_{f}\right)\left(c_{s} p_{f}-c_{T} \Delta T_{s}\right) \\
\Delta s^{s} & =\frac{c_{T}}{c_{\mathrm{DS}}} \operatorname{tr} \boldsymbol{\epsilon}-\left(\xi_{f}-n_{f}\right) c_{T} p_{f}+\frac{\rho^{s} C_{s}^{(v)}}{T^{0}} \Delta T_{s}
\end{aligned}
$$

in which $C_{s}^{(v)}[\mathrm{J} / \mathrm{kg} . \mathrm{K}]$ is the intrinsic specific heat capacity of the solid, i.e. per unit mass of solid, at constant volume and fluid pressure.

The thermodynamic potentials of the fluid are built separately. Assuming that the hydraulic compressibility, the thermal expansion coefficient and the heat capacity are constant, the change of the apparent entropy of the fluid is expressed as [26],

$$
\Delta s^{f}=-n_{f} c_{f T} \Delta p_{f}+\frac{\rho^{f} C_{f}^{(p)}}{T_{f}} \Delta T_{f},
$$

where $C_{f}^{(p)}[\mathrm{J} / \mathrm{kg} . \mathrm{K}]$ is the intrinsic specific heat capacity of the fluid, i.e. per unit mass of the fluid, at constant pressure. The fluid enthalpy $H_{f}$ and the fluid density $\rho_{f}$ depend on the pressure and temperature only, so that the enthalpy gradient has the format,

$$
\nabla H_{f}=\left(1-T_{f} c_{f T}\right) \frac{\nabla p_{f}}{\rho_{f}}+C_{f}^{(p)} \nabla T_{f}
$$

while the change of the intrinsic mass density,

$$
\frac{1}{\rho_{f}} \frac{\mathrm{d}^{f} \rho_{f}}{\mathrm{~d} t}=c_{f H} \frac{\mathrm{d}^{f} p_{f}}{\mathrm{~d} t}-c_{f T} \frac{\mathrm{d}^{f} T_{f}}{\mathrm{~d} t},
$$

expresses in terms of the hydraulic compressibility $c_{f H}[1 / \mathrm{Pa}]$ and of the coefficient of thermal expansion $c_{f T}[1 / \mathrm{K}]$,

$$
c_{f H}=\left.\frac{1}{\rho_{f}} \frac{\mathrm{d}^{f} \rho_{f}}{\mathrm{~d} p_{f}}\right|_{T_{f}}, \quad c_{f T}=-\left.\frac{1}{\rho_{f}} \frac{\mathrm{d}^{f} \rho_{f}}{\mathrm{~d} T_{f}}\right|_{p_{f}} .
$$

Fluid flow and heat diffusion are defined by uncoupled Darcy's law and Fourier's law respectively. Darcy's law of seepage through the solid skeleton,

$$
\mathbf{j}_{f}=-\frac{k_{f}}{\mu_{f}}\left(\nabla p_{f}-\rho_{f} \mathbf{g}\right),
$$

expresses in terms of the macroscopic permeability of the fracture network $k_{f}\left[\mathrm{~m}^{2}\right]$ and of the dynamic viscosity $\mu_{f}[\mathrm{~Pa} . \mathrm{s}]$, while Fourier's law of heat transfer through phase $k$,

$$
\mathbf{q}_{k}=-n_{k} \Lambda_{k} \nabla T_{k}, \quad k=s, f .
$$


requires the intrinsic thermal conductivity $\Lambda_{k}[\mathrm{~W} / \mathrm{m} . \mathrm{K}]$.

Finally, the rate of energy transfer from the solid to the fluid,

$$
\hat{e}_{s f}=\kappa_{s f}\left(T_{s}-T_{f}\right),
$$

is simply proportional to the temperature differential. It agrees with 'Newton's law of cooling' which states that the rate of temperature decrease of a body immersed in a fluid is at all times proportional to the body-fluid temperature difference. Satisfaction of the thermodynamics of irreversible processes requires the specific inter-phase heat transfer coefficient between the solid phase and the fluid $\kappa_{s f} \geq 0\left[\mathrm{~W} / \mathrm{m}^{3} . \mathrm{K}\right]$ to be positive.

\section{Finite element discretization}

The finite element formulation considers the displacement vector $\mathbf{u}$, fluid pressure $p_{f}$, temperature of the solid skeleton $T_{s}$ and temperature of the fluid $T_{f}$ as primary unknowns. The finite element code written in FORTRAN has been developed as part of this work and the model predictions have been verified against analytical and numerical models as presented in [26].

\subsection{The semi-discrete equations}

Within the generic element $e$, the primary unknowns,

$$
\mathbf{u}=\mathbf{N}_{\mathbf{u}} \mathbf{u}^{e}, \quad p_{f}=\mathbf{N}_{p} \mathbf{p}_{f}^{e}, \quad T_{k}=\mathbf{N}_{T} \mathbf{T}_{k}^{e}, \quad k=s, f,
$$

are interpolated in terms of nodal values through the respective interpolation functions $\mathbf{N}_{\mathbf{u}}, \mathbf{N}_{p}, \mathbf{N}_{T}$. Multiplying the field equations (1) by the virtual fields $\delta \mathbf{u}, \delta p, \delta T_{s}$ and $\delta T_{f}$, and integrating by parts over the body $V$, provides the weak form of the problem,

$$
\begin{aligned}
\int_{V} \nabla(\delta \mathbf{u}): \boldsymbol{\sigma}-\delta \mathbf{u} \cdot \rho \mathbf{g} \mathrm{d} V & =\int_{\partial V} \delta \mathbf{u} \cdot \boldsymbol{\sigma} \cdot \hat{\mathbf{n}} \mathrm{d} S, \\
\int_{V} \nabla(\delta p) \cdot \mathbf{j}_{f}-\delta p \mathcal{J}_{f} \mathrm{~d} V & =\int_{\partial V} \delta p \mathbf{j}_{f} \cdot \hat{\mathbf{n}} \mathrm{d} S, \\
\int_{V} \nabla\left(\delta T_{s}\right) \cdot \mathbf{q}_{s}-\delta T_{s} \mathcal{Q}_{s} \mathrm{~d} V & =\int_{\partial V} \delta T_{s} \mathbf{q}_{s} \cdot \hat{\mathbf{n}} \mathrm{d} S, \\
\int_{V} \nabla\left(\delta T_{f}\right) \cdot \mathbf{q}_{f}-\delta T_{f} \mathcal{Q}_{f} \mathrm{~d} V & =\int_{\partial V} \delta T_{f} \mathbf{q}_{f} \cdot \hat{\mathbf{n}} \mathrm{d} S,
\end{aligned}
$$

where $\hat{\mathbf{n}}$ is the unit outward normal to the boundary $\partial V$. A standard Galerkin procedure is adopted for the balances of momentum $(15)_{1}$, the balance of mass of the fluid $(15)_{2}$ and the balance of energy of the solid $(15)_{3}$. On the other hand, the streamline-upwind/Petrov-Galerkin procedure (SUPG) devised by Brooks and Hughes [27] is adopted for the treatment of the convective term $\rho^{f} \mathbf{v}_{\text {conv }} \cdot \nabla H_{f}$ in the balance of energy of the fluid phase $(15)_{4}$. The convective velocity $\mathbf{v}_{\text {conv }}$ is identified as the diffusion velocity $\mathbf{v}_{f}-\mathbf{v}_{s}$. The virtual field $\delta T_{f}$ is interpolated via the function $\mathbf{W}_{T}$,

$$
\mathbf{W}_{T}=\mathbf{N}_{T}+\tau_{\mathrm{SUPG}} \mathbf{v}_{\mathrm{conv}} \cdot \nabla \mathbf{N}_{T},
$$

in which the perturbation is weighted by the time-like parameter $\tau_{\mathrm{SUPG}}$. The modified interpolation function $\mathbf{W}_{T}$ applies to the whole differential equation. This procedure ensures that consistency is enforced from the beginning which distinguishes the SUPG method from the artificial diffusion method [27, 28]. By summing the elementary contributions over the total number of elements nel, the semi-discretized form of eqn (15) 4 may be transformed to,

$$
\sum_{e=1}^{\text {nel }} \int_{V^{e}}\left(\delta \mathbf{T}_{f}^{e}\right)^{\mathrm{T}}\left(\left(\nabla \mathbf{N}_{T}\right)^{\mathrm{T}} \mathbf{q}_{f}-\left(\mathbf{W}_{T}\right)^{\mathrm{T}} \mathcal{Q}_{f}\right) \mathrm{d} V^{e}=\sum_{e=1}^{\text {nel }} \int_{\partial V^{e}}\left(\delta \mathbf{T}_{f}^{e}\right)^{\mathrm{T}}\left(\mathbf{N}_{T}\right)^{\mathrm{T}} \mathbf{q}_{f} \cdot \hat{\mathbf{n}} \mathrm{d} S^{e} .
$$


The stabilization contribution is restricted to the element interiors, since the conductivity $\Lambda_{f}$ is isotropic and bi-linear elements are used [27]. The stabilization parameter $\tau_{\text {SUPG }}$ devised by Tezduyar and Osawa [29],

$$
\tau_{\mathrm{SUPG}}=\left(\frac{1}{\tau_{S 1}^{2}}+\frac{1}{\tau_{S 2}^{2}}+\frac{1}{\tau_{S 3}^{2}}\right)^{-\frac{1}{2}}
$$

is built from the three times $\tau_{S 1}, \tau_{S 2}, \tau_{S 3}$ associated respectively with convection-dominated, transientdominated and diffusion-dominated flows,

$$
\tau_{S 1}=\frac{h}{2\left|\mathbf{v}_{\text {conv }}\right|}, \quad \tau_{S 2}=\frac{\Delta t}{2}, \quad \tau_{S 3}=\frac{h^{2}}{4 \alpha_{T, f}}=\operatorname{Pe}_{\mathrm{g}} \tau_{S 1} .
$$

Here $\alpha_{T, f}$ is the thermal diffusivity of the fluid and $h$ is the 'element length' in the direction of the flow defined as [29]

$$
h=2\left|\mathbf{v}_{\text {conv }}\right|\left(\sum_{a=1}^{n_{\mathrm{ne}}}\left|\mathbf{v}_{\mathrm{conv}} \cdot \nabla \mathbf{N}_{T}^{a}\right|\right)^{-1},
$$

and $n_{\mathrm{ne}}$ is the number of element nodes. Note that the definition of $\tau_{\mathrm{SUPG}}$ in eqn (18) does not depend of the solution $T_{f}$ but is dependent on the velocity $\mathbf{v}_{\text {conv }}$ and on the time step $\Delta t$. The stabilization parameter (18) reduces to the exact form for 1-dimensional and steady state problems $\tau_{S 1}=h / 2\left|\mathrm{v}_{\text {conv }}\right|$ if the grid Péclet number $\mathrm{Pe}_{\mathrm{g}}=h\left|\mathrm{v}_{\text {conv }}\right| / 2 \alpha_{T, f}$ is high, that is for a convection-dominated flow [27]. The term $\tau_{S 2}$ smooths the response at early times. The diffusion-dominated limit $\tau_{S 3}$ is three times the value of the latter reference.

The resulting non-linear first-order semi-discrete equations for the unknown vector $\mathbb{X}$,

$$
\mathbb{X}=\left[\begin{array}{llll}
\mathbf{u} & \mathbf{p}_{f} & \mathbf{T}_{s} & \mathbf{T}_{f}
\end{array}\right]^{\mathrm{T}}
$$

with maximum nodal length $=$ dimension of space +3 , imply the residual $\mathbb{R}$,

$$
\mathbb{R}=\mathbb{F}^{\text {grav }}+\mathbb{F}^{\text {surf }}(\mathbb{S}, \mathbb{X})-\mathbb{F}^{\text {int }+ \text { conv }}\left(\mathbb{X}, \frac{\mathrm{d} \mathbb{X}}{\mathrm{d} t}\right)=\mathbb{O}
$$

to vanish. Here $\mathbb{F}^{\text {int+conv }}$ is the vector that contains the internal forces together with the convective contributions including the SUPG stabilization, appearing on the left-hand-side of eqns (15), $\mathbb{F}^{\text {surf }}$ is the vector of surface loadings denoted collectively $\mathbb{S}$ and $\mathbb{F}^{\text {grav }}$ is the vector contributed by gravity (Appendix A). The residual vector includes terms associated with the four physical phenomena of interest,

$$
\mathbb{R}=\left[\begin{array}{llll}
\mathbf{R}_{\mathbf{u}} & \mathbf{R}_{p_{f}} & \mathbf{R}_{T_{s}} & \mathbf{R}_{T_{f}}
\end{array}\right]^{\mathrm{T}}
$$

\subsection{Time integration}

The semi-discrete equations are integrated through a generalized trapezoidal rule defined by a scalar $\alpha \in] 0,1]$. At step $n+1$, the equations are enforced at time $t_{n+\alpha}=t_{n}+\alpha \Delta t$, with $\Delta t=t_{n+1}-t_{n}$, namely,

$$
\mathbb{R}_{n+\alpha}=\mathbb{F}^{\text {grav }}+\mathbb{F}^{\text {surf }}\left(\mathbb{S}_{n+\alpha}, \mathbb{X}_{n+\alpha}\right)-\mathbb{F}^{\text {int+conv }}\left(\mathbb{X}_{n+\alpha}, \mathbb{V}_{n+\alpha}\right)=\mathbb{O}
$$

In the above relation, $\mathbb{Z}=\mathbb{S}, \mathbb{X}, \mathbb{V}$ is defined as $\mathbb{Z}_{n+\alpha}=(1-\alpha) \mathbb{Z}_{n}+\alpha \mathbb{Z}_{n+1}$, and $\mathbb{X}_{n+1}$ and $\mathbb{V}_{n+1}$ are approximations of $\mathbb{X}\left(t_{n+1}\right)$ and $(\mathrm{d} \mathbb{X} / \mathrm{d} t)\left(t_{n+1}\right)$, respectively.

The system (24) is solved iteratively by an explicit-implicit operator split, namely at iteration $i+1$,

$$
\mathbb{R}_{n+\alpha}^{i+1}=\mathbb{F}_{\mathrm{E}}^{\text {grav }}+\mathbb{F}_{\mathrm{E}}^{\text {surf }}\left(\mathbb{S}_{n+\alpha}, \mathbb{X}_{n+\alpha}^{i}\right)-\mathbb{F}_{\mathrm{I}}^{\text {int }+\operatorname{conv}}\left(\mathbb{X}_{n+\alpha}^{i+1}, \mathbb{V}_{n+\alpha}^{i+1}\right)=\mathbb{O}
$$

The global iteration process uses a full Newton-Raphson procedure. The Newton direction $\Delta \mathbb{V}$ is sought by setting eqn (25) to zero,

$$
\mathbb{C}(\alpha \Delta \mathbb{V})=\mathbb{R}_{n+\alpha}^{i},
$$


in which the effective diffusion matrix $\mathbb{C}$ can be expressed in terms of the global diffusion matrix $\mathbb{D}=\mathbb{D}(\mathbb{X}, \mathbb{V})$ and of the global stiffness-convection matrix $\mathbb{K}=\mathbb{K}(\mathbb{X}, \mathbb{V})$,

$$
\mathbb{C}=\mathbb{D}+\mathbb{K} \alpha \Delta t .
$$

The global diffusion and stiffness-convection matrices are obtained by assembling the element contributions which have the following block structure,

$$
\mathbb{D}^{e}=\left[\begin{array}{cccc}
0 & 0 & 0 & 0 \\
\mathbf{D}_{p_{f} \mathbf{u}}^{e} & \mathbf{D}_{p_{f} p_{f}}^{e} & \mathbf{D}_{p_{f} T_{s}}^{e} & \mathbf{D}_{p_{f} T_{f}}^{e} \\
\mathbf{D}_{T_{s} \mathbf{u}}^{e} & \mathbf{D}_{T_{s} p_{f}}^{e} & \mathbf{D}_{T_{s} T_{s}}^{e} & 0 \\
0 & { }^{*} \mathbf{D}_{T_{f} p_{f}}^{e} & 0 & { }^{*} \mathbf{D}_{T_{f} T_{f}}^{e}
\end{array}\right] ; \quad \mathbb{K}^{e}=\left[\begin{array}{cccc}
\mathbf{K}_{\mathbf{u u}}^{e} & \mathbf{K}_{\mathbf{u} p_{f}}^{e} & \mathbf{K}_{\mathbf{u} T_{s}}^{e} & 0 \\
0 & \mathbf{K}_{p_{f} p_{f}}^{e} & 0 & 0 \\
0 & 0 & \mathbf{K}_{T_{s} T_{s}}^{e} & \mathbf{K}_{T_{s} T_{f}}^{e} \\
0 & { }^{*} \mathbf{K}_{T_{f} p_{f}}^{e} & { }^{*} \mathbf{K}_{T_{f} T_{s}}^{e} & { }^{*} \mathbf{K}_{T_{f} T_{f}}^{e}
\end{array}\right]
$$

The detailed expressions of these matrices are given in Appendix B. Convection terms that require a special treatment are highlighted with the superscript *

The four-node element (Q4) is used to interpolate all unknowns. For all simulations, element sizes of $10 \times 10 \mathrm{~m}^{2}$ are used. The number of integration points is equal to two (for each space dimension), for all matrices and all residuals including the convective contributions.

Each component of the residual vector $\mathbb{R}$ is scaled by a reference value: $R_{u \text {,ref }}=75.0 \times 10^{6} \mathrm{~N}$ for the balance of momentum contributions, $\mathrm{R}_{p_{f} \text {,ref }}=1.0 \times 10^{-6} \mathrm{~m}^{3} / \mathrm{s}$ for the balance of mass contribution and $\mathrm{R}_{T_{s}, \text { ref }}=\mathrm{R}_{T_{f}, \text { ref }}=1.26 \mathrm{~W}$ for the balance of energy contributions. $\mathrm{R}_{u \text {,ref }}$ represents the overburden stress, $\mathrm{R}_{p_{f} \text {,ref }}$ the maximum fluid flow and $\mathrm{R}_{T_{s} \text {,ref }}=\mathrm{R}_{T_{f} \text {,ref }}$ the maximum heat flow; all four quantities referring to a unit area of one square meter. The norm of the residual at each iteration $i$ is then calculated by summing the neq contributions (neq $=$ number of equations),

$$
\left|\mathbb{R}^{i}\right|=\sum_{a=1}^{\text {neq }}\left|\frac{\mathbf{R}_{a}}{\mathrm{R}_{a, \text { ref }}}\right| .
$$

Iterations are stopped when the tolerances below involving both the overall scaled residual and unknowns are satisfied:

$$
\begin{aligned}
& \text { - }\left|\mathbb{R}^{i}\right| /\left|\mathbb{R}^{1}\right|<0.001 ; \\
& \text { - }\left|x^{i}-x^{i-1}\right| /\left|x^{i}\right|<0.001, \quad \text { for } x=u_{j}, p_{f}, T_{s}, T_{f} .
\end{aligned}
$$

The average number of Newton-Raphson iterations per time step has been observed to remain around 3 .

\subsection{Characteristic times}

The time integration parameter $\alpha$ is taken equal to $2 / 3$. Conditional stability characterizes non-linear transient convective-diffusive problems discretized with a full Newton-Raphson scheme [30]. The time step $\Delta t$ is increased by fits and starts in the range $\left[\Delta t_{\min }, \Delta t_{\max }\right]$ in order to keep the computation time within acceptable limits. The lower bound $\Delta t_{\min }$ is associated with the fastest diffusion time, that is the hydraulic diffusion of the fluid and is maintained constant until hydraulic steady state is reached. $\Delta t_{\max }$ is obtained with respect to the slowest remaining diffusion, that is the thermal diffusion of the solid. The hydraulic diffusivity $\alpha_{H}$ depends on the seepage and mechanical properties,

$$
\alpha_{H}=\frac{k_{f}}{\mu_{f}} \frac{2 \mu_{\mathrm{DS}}(1-\nu)}{1-2 \nu} \frac{A^{2}\left(1+\nu_{u}\right)^{2}(1-2 \nu)}{9\left(1-\nu_{u}\right)\left(\nu_{u}-\nu\right)},
$$

in which $A$ is the Skempton coefficient,

$$
\frac{1}{A}=1+n_{f} \frac{c_{f H}-c_{s}}{c_{\mathrm{DS}}-c_{s}},
$$

and $\nu_{u}$ is the undrained Poisson's ratio,

$$
\nu_{u}=\frac{3 \nu+A(1-2 \nu)\left(1-c_{s} / c_{\mathrm{DS}}\right)}{3-A(1-2 \nu)\left(1-c_{s} / c_{\mathrm{DS}}\right)} .
$$


The thermal diffusivities $\alpha_{T, s}$ and $\alpha_{T, f}$ through the solid and the fluid, respectively, involve only thermal properties,

$$
\alpha_{T, s}=\frac{n_{s} \Lambda_{s}}{\rho^{s} C_{s}^{(v)}}, \quad \alpha_{T, f}=\frac{n_{f} \Lambda_{f}}{\rho^{f} C_{f}^{(p)}} .
$$

Heat transport in the fluid phase is dominated by convection due to the high permeability of the fracture network whereas heat transport in the solid phase is controlled by diffusion. The characteristic time associated with convection at speed of $\mathrm{v}_{f, z}$ is proportional to the distance traveled $L$,

$$
t_{\mathrm{conv}}=\frac{L}{\mathrm{v}_{f, z}} .
$$

On the other hand, the characteristic times associated with seepage and with thermal diffusion in phase $k$ depend on the square of the distance traveled $L$,

$$
t_{\text {Hdiff }}=\frac{L^{2}}{\alpha_{H}}, \quad t_{\text {Tdiff }, k}=\frac{L^{2}}{\alpha_{T, k}} .
$$

In the tests reported in Sections 5 and 6 , and in geothermal tests in general, the fluid velocity $\mathrm{v}_{f, z}$ ranges from $O\left(10^{-6}\right) \mathrm{m} / \mathrm{s}$ to $O\left(10^{-3}\right) \mathrm{m} / \mathrm{s}$, the hydraulic diffusivity of water typically of the order $O\left(10^{1}\right) \mathrm{m}^{2} / \mathrm{s}$ is much larger than the thermal diffusivity of the solid about $O\left(10^{-6}\right) \mathrm{m}^{2} / \mathrm{s}$ which is itself an order of magnitude larger than the thermal diffusivity of water $O\left(10^{-7}\right) \mathrm{m}^{2} / \mathrm{s}$. Consequently, over a diffusion length of $L=230 \mathrm{~m}$ separating the injection and production wells (see Sections 5 and 6 ), the characteristic times associated with convection and diffusion, through the solid phase and the fluid phase, range as follows,

$$
\begin{aligned}
& t_{\text {Hdiff }} \approx 1 \text { hour, } \\
& t_{\text {conv }} \in[2.66 \text { days, } 7.31 \text { years }], \\
& t_{\text {Tdiff }, s} \approx 1,500 \text { years, } \\
& t_{\text {Tdiff }, f} \approx 10,000 \text { years. }
\end{aligned}
$$

Hence, in absence of heat transfer across the solid-fluid interface, the rate of the temperature propagation, and its impacts on pressure and displacement fields, can be up to $10^{5}$ times faster in the convection-dominated fracture network than in the diffusion-dominated solid skeleton. Therefore local thermal equilibrium is unlikely to be established before the late period of the circulation test, and the overall thermo-hydromechanical behavior of the mixture is unlikely to be accurately modeled if local thermal non-equilibrium between the solid phase and the fluid phase is not accounted for.

Remark 3.1. According to (34), the stabilization parameter for convection-dominated flows $\tau_{S 1}$, eqn (19), can be interpreted as the time required for a particle to be convected over half the length of the element.

\section{HDR reservoir analysis}

The fully coupled thermo-hydro-mechanical model is used in the subsequent sections to investigate the thermal drawdown of a generic hot dry rock reservoir in local thermal non-equilibrium. Information on the geometry, initial and boundary conditions considered are provided first.

\subsection{Geometry of the HDR reservoir}

An artificially fractured reservoir with horizontal injection and production wells is considered (Fig. 1). The simulations assume a plane strain analysis. The finite element mesh includes half of the reservoir and a portion of the surrounding low permeability rock formation. The HDR fractured reservoir is idealized by a single porosity saturated medium. The material properties and constitutive equations characterizing the processes involved are described in Sect. 2.2. 


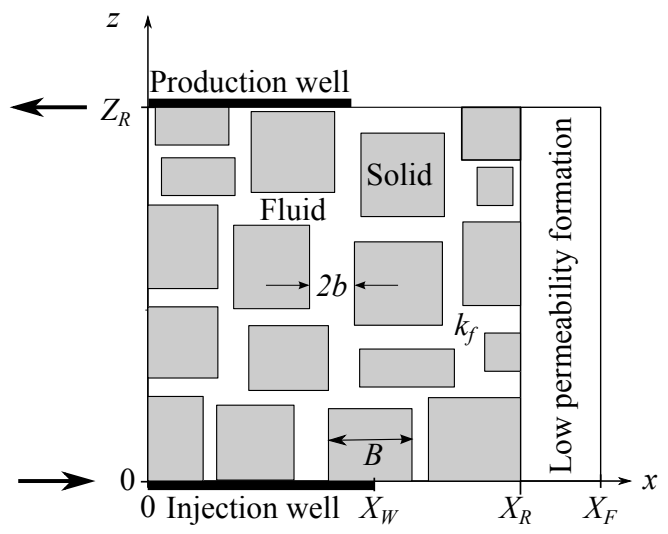

Figure 1: Representation of a generic HDR reservoir (not at scale). The permeability $k_{f}$ of the reservoir depends on the average fracture spacing $B$ and on the average fracture aperture $2 b$. The simulations assume a plane strain analysis, in the $x-z$ plane, and symmetry with respect to $z$-axis.

Adding the surrounding low permeability formation in the analysis allows to assess the influence of the external heat supply which is presented in Sect.5.5. The width of this surrounding domain $X_{F}-X_{R}$ is chosen so that the characteristic time of heat diffusion, eqn (35), is greater than the life-time of the reservoir $(\approx 20$ years $)$. The formation is endowed with the same material properties as the reservoir except for the permeability.

The injection and the production wells are located at the bottom and at the top of the reservoir, respectively. The length of the wells is a key parameter that governs the flow path and the heat transfer between the rock and the fluid. Two setups are considered:

1. in one case, the wells penetrate horizontally into the entire reservoir, that is $X_{W}=X_{R}$. Consequently, the velocity of the fluid $\mathbf{v}_{f}$ is vertical and uniform between the two wells, Fig. 6, left;

2. in the second case, the wells penetrate into one third of the reservoir, leading to non-uniform velocity field, Fig. 6, right.

\subsection{The specific inter-phase heat transfer coefficient $\kappa_{s f}$}

The coefficient of specific inter-phase heat transfer $\kappa_{s f}\left[\mathrm{~W} / \mathrm{m}^{3} . \mathrm{K}\right]$ controls the rate at which the two phase system (solid-fluid) reaches thermal equilibrium. The higher this coefficient, the faster thermal equilibrium is reached. The coefficient of specific inter-phase heat transfer is defined by the product of the solid-fluid specific surface $S_{s f}^{s}\left[\mathrm{~m}^{2} / \mathrm{m}^{3}\right]$ with the coefficient of solid-fluid heat transfer $h_{s f}\left[\mathrm{~W} / \mathrm{m}^{2} . \mathrm{K}\right]$,

$$
\kappa_{s f}=S_{s f}^{s} \times h_{s f}
$$

For a square block of size $B$ bordered by a fracture fluid of width $b$, the volume fractions of the solid and fluid are equal to

$$
n_{s}=\frac{B^{2}}{(B+2 b)^{2}}, \quad n_{f}=\frac{4 b(B+b)}{(B+2 b)^{2}} .
$$

The specific surface $S_{s f}^{s}$ is the total surface area of the interstitial voids divided by the total volume of the medium,

$$
S_{s f}^{s}=\frac{4 B}{(B+2 b)^{2}} .
$$

For $2 b \ll B$, the specific surface area simplifies to

$$
S_{s f}^{s}=\frac{2 n_{f}}{2 b}=\frac{4}{B} .
$$


$S_{s f}^{s}$ vanishes if the fracture porosity tends to zero $n_{f} \rightarrow 0$, for a constant aperture $2 b$, or if the fissure spacing tends towards infinity $B \rightarrow \infty$. A discussion on the theoretical formulation of the specific surface area (40) is provided in Appendix C.

The coefficient of solid-fluid heat transfer $h_{s f}$ (also known as the particle-to-fluid heat transfer coefficient) depends of the nature of the solid, the nature of the fluid and the dominant regime of heat transport. By assuming that the effect of convection in the fracture fluid phase (perpendicular to the solid-fracture interface) is negligible, the coefficient of solid-fluid heat transfer may be quantitatively characterized by the sum of the thermal resistances of the two phases in series [31],

$$
\frac{1}{h_{s f}}=\frac{2 b}{2 n_{f} \Lambda_{f}}+\frac{B}{2 n_{s} \Lambda_{s}}
$$

The general form of the specific coefficient of heat transfer between the solid and the fluid phase $\kappa_{s f}$ is non-linearly related to $B$ and $b$ through eqs (37), (39), (41). This general form should be used when the ratio between the fracture aperture and the fracture spacing is in constant evolution, for example during comminution. On the other hand, in geothermal applications, the fracture width is much smaller than the fracture spacing, namely $2 b \ll B$. Then the specific coefficient of heat transfer between the solid and the fluid phase is linearly related to $B^{-2}$,

$$
\kappa_{s f}=\frac{8}{B^{2}} \frac{n_{s} \Lambda_{s} \times 2 \Lambda_{f}}{n_{s} \Lambda_{s}+2 \Lambda_{f}} .
$$

The thermal conductivities of the two phases are involved. For a geothermal reservoir, typical values entail $n_{s} \approx 1, \Lambda_{s}=2.71 \mathrm{~W} / \mathrm{m} . \mathrm{K}$ and $\Lambda_{f}=0.6 \mathrm{~W} / \mathrm{m} . \mathrm{K}$, and the two phases are seen to contribute to the overall conductivity. Alternatively, assuming $n_{s} \Lambda_{s} \ll 2 \Lambda_{f}$, the above relation would reduce to,

$$
\kappa_{s f}=8 \frac{n_{s} \Lambda_{s}}{B^{2}}
$$

where the two phases are not treated symmetrically, à la Warren and Root [32], with emphasis on the most insulating material.

Correlations of the solid-fluid heat transfer coefficient $h_{s f}$ have been proposed in the literature with the Nusselt number [33] and with a capillary tube model [34]. Few experimental works have investigated the magnitude of the solid-fluid heat transfer coefficient $h_{s f}[35,36]$. Instead, here, a sensitivity analysis is performed to determine the threshold value that separates local thermal equilibrium from the local thermal non-equilibrium [37, 38]. A calibration of the model, and hence of the specific inter-phase heat transfer coefficient, with help of data provided from the Fenton Hill HDR reservoir is proposed in Sect. 6.

\subsection{Initial conditions}

Prior to the circulation test, the reservoir is assumed to be in local thermal equilibrium, and the solid and fluid have identical temperatures $T_{s}^{0}=T_{f}^{0}=T^{0}$. The geothermal gradient is neglected since the height of the reservoir is small with respect to its average depth $H$. The initial pressure of the fluid $p_{f}^{0}=\rho_{f} g H$ is assumed proportional to the depth of the point of interest $H$, the fluid density $\rho_{f}$ and the gravity $g$. The initial stress state is due to the overburden stress and to the lateral earth stress.

\subsection{Boundary conditions}

The thermal, hydraulic and mechanical boundary conditions are sketched in Fig. 2.

The injection temperature $T_{\text {inj }}$ is applied to all the phases along the injection well. The outlet temperature $T_{f \text {,out }}$ along the producing well is an output of the simulations. The remaining horizontal and vertical boundaries are thermally insulated from the surroundings, that is $\mathbf{q}_{s} \cdot \hat{\mathbf{n}}=\mathbf{q}_{f} \cdot \hat{\mathbf{n}}=0$, $\hat{\mathbf{n}}$ being the local unit outward normal.

The injection and production pressures, $p_{f, \text { inj }}$ and $p_{f, \text { out }}$ respectively, are both specified along the injection and production wells. The remaining boundaries are hydraulically impermeable, i.e. $\mathbf{j}_{f} \cdot \hat{\mathbf{n}}=0$. 
Thermal BC

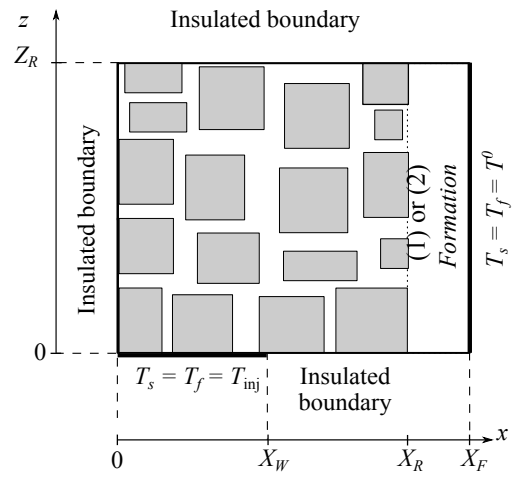

Hydraulic BC

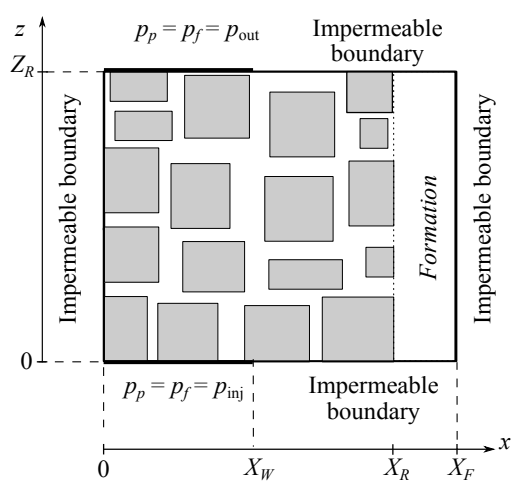

Mechanical BC

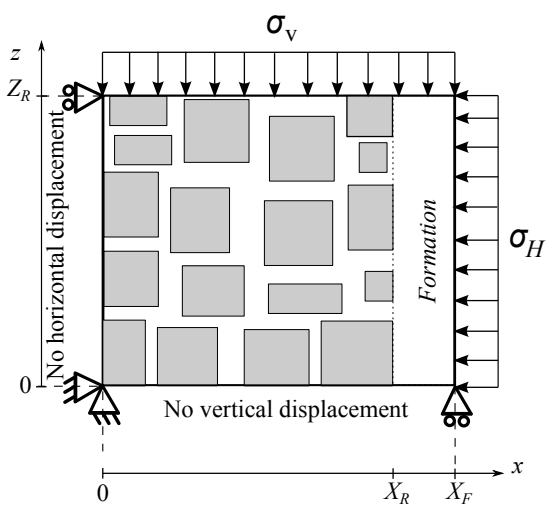

Figure 2: Thermal, hydraulic and mechanical boundary conditions.

The vertical stress $\sigma_{z}=\sigma_{z}^{0}$ remains constant along the top boundary $z=Z_{R}$, and so does the horizontal stress $\sigma_{x}=\sigma_{x}^{0}$ along the lateral boundary $x=X_{F}$. The displacements on the other boundaries are specified, $u_{x}(x=0, z)=0$ and $u_{z}(x, z=0)=0$.

At the contact between the reservoir and the surrounding rock $x=X_{R}$, two types of interactions are considered: (1) the reservoir is thermally insulated from the rock formation; (2) the reservoir exchanges heat with the formation which remains at the initial temperature $T^{0}$, namely $T_{s}=T_{f}=T^{0}$ along $x=X_{F}$.

\section{The double-step pattern of thermal depletion in LTNE}

This section focuses on the influence of the LTNE assumption on the time profiles of the fluid temperature (at the producing well). The reservoir response in terms of fluid pressure, displacement and effective stress will be presented in Sect. 6 .

In the context of a fluid saturated fractured medium, reservoir performances can be expressed in a general form in terms of the fracture porosity $n_{f}$ and two dimensionless parameters [4]. These parameters reflect the overwhelming importance of the characteristic lengths of the fracture network, namely the average aperture $2 b$ and the average spacing $B$.

(1) The dimensionless temperature $T_{D}$ built from the outlet fluid temperature $T_{f \text {,out }}$, the initial temperature $T^{0}$ and the injection temperature $T_{\mathrm{inj}}$,

$$
T_{D}=\frac{T^{0}-T_{f, \text { out }}}{T^{0}-T_{\mathrm{inj}}}
$$

ranges between $0\left(T_{f, \text { out }}=T^{0}\right)$ at early time and $1\left(T_{f, \text { out }}=T_{\text {inj }}\right)$ ultimately.

(2) Adapted from the work of Minkowycz et al. [37] and Nield et al. [38], the dimensionless parameter $\eta_{D}$ that serves to delineate LTE and LTNE,

$$
\eta_{D}=\frac{1}{n_{f}} \frac{\mathrm{Sp}}{\mathrm{Pe}}
$$

is defined in terms of the fracture porosity $n_{f}$, of a modified Sparrow number Sp which measures the relative weights of heat transfer across the system and heat conduction through the porous medium, and of the Péclet number Pe, which measures the relative weights of convection and thermal conduction,

$$
\mathrm{Sp}=\frac{\kappa_{s f} Z_{R}^{2}}{n_{s} \Lambda_{s}+n_{f} \Lambda_{f}}, \quad \mathrm{Pe}=\frac{Z_{R} \mathrm{v}^{\infty}}{\alpha_{T, f}} .
$$

The general form of the dimensionless LTNE parameter $\eta_{D}$ expresses in terms of the thermal properties of the porous medium, $\Lambda_{s}, \Lambda_{f}$ and $\alpha_{T, f}$, the fluid porosity $n_{f}$, the diffusion-convection length $Z_{R}$, the 

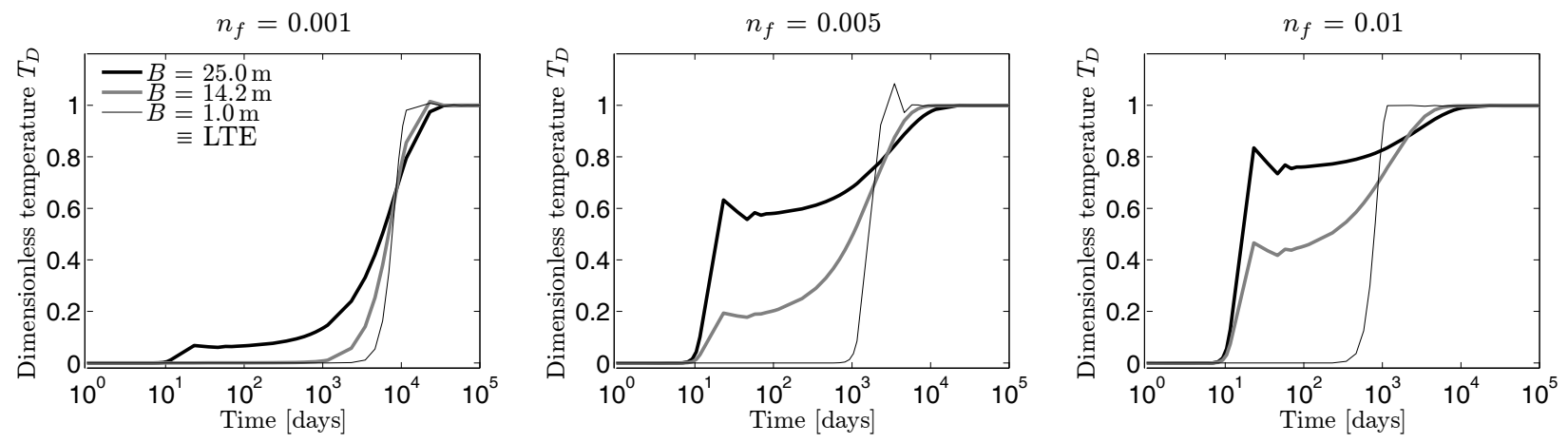

Figure 3: Dimensionless temperature outlet $T_{D}$ as a function of time for three porosities $n_{f}$ and three fracture spacings $B$. All results are for $Z_{R}=230 \mathrm{~m}, \mathrm{v}^{\infty}=2 \times 10^{-4} \mathrm{~m} / \mathrm{s}$ and thermal properties from Table 2 . In LTNE, time profiles display a double-step pattern, whereas in LTE, time profiles display a continuous pattern. The thresholds between LTE and LTNE are associated with the fracture spacings $B_{T}=6 \mathrm{~m}$ (left), $B_{T}=2.5 \mathrm{~m}$ (middle) and $B_{T}=2 \mathrm{~m}$ (right). The corresponding dimensionless values of $\eta_{D}$ are equal respectively to 11, 13 and 10. Therefore, LTE is associated with $\eta_{D}$ larger than, say 13 , while values of $\eta_{D}$ smaller than 13 requires a LTNE analysis. The late overshooting oscillations are numerical artifacts due to an imperfect damping of the convective contribution, see Remark 5.1.

aperture of the fracture network $2 b$ and the steady state fluid velocity $\mathrm{v}^{\infty}$ through eqns (40) and (42). For $n_{s} \Lambda_{s} \gg n_{f} \Lambda_{f}$ and for $n_{s} \approx 1$, the above dimensionless LTNE parameter simplifies to

$$
\eta_{D}=\frac{1}{n_{f}} \frac{16 \Lambda_{f}}{\Lambda_{s}+2 \Lambda_{f}} \frac{Z_{R} \alpha_{T, f}}{\mathrm{v}^{\infty} B^{2}} .
$$

Of prime interest for the economical success of a HDR reservoir is the knowledge of the time profile of the temperature drawdown $T_{f \text {, out }}$ for representative steady state fluid velocities $\mathrm{v}^{\infty}$. The fracture porosity $n_{f}$ for igneous and metamorphic rocks ranges from 0.0005 to $0.01[3,5,13]$.

First, thermal drawdown curves are observed to show a single-step pattern for small fracture spacings $B$ and double-step patterns for large fracture spacings (Sect. 5.1). The threshold values between LTNE and LTE are sought in terms of the fracture spacing $B_{T}$ for an average steady state velocity and several fluid porosities in Sect. 5.2. These thresholds define the onset of LTNE in which thermal drawdown curves change from a single-step pattern to a double-step pattern. A hyperbolic dependency between $\left(B_{T}\right)^{2}$ and $n_{f}$ is observed which matches with eqn (47) for $n_{f}$ over the range of interest. Sect. 5.3 addresses the influence of the pumping rate on the fracture spacing thresholds $B_{T}$. The hyperbolic relation between $\left(B_{T}\right)^{2}$ and $\mathrm{v}^{\infty}$ suggested by eqn (47) is well recovered by the finite element simulations.

Attention is restricted to a uniform and vertical flow, while the reservoir is insulated from the rock formation. Departures from this setting are considered in Sects.5.4 and 5.5. The issue of fluid loss is ignored throughout and will be addressed in a subsequent paper [39].

\subsection{Single-step versus double-step patterns}

A change of time profile is observed as the fracture spacing $B$ increases. Time profiles for small $B$ are clearly dominated by convection and display a characteristic single-step pattern. Conversely, the time profiles for large $B$ display three stages and typical double-step patterns.

(1) In the first stage, the strong increase of the dimensionless temperature corresponds to the abrupt propagation of the injection temperature dominated by convection. This effect is mostly attributed to the difference in characteristic times between forced convection in the fracture network and diffusion of heat in the solid matrix. Accordingly for each fracture porosity $n_{f}$, the magnitude of the dimensionless temperature $T_{D}$ at the end of this first stage is increasing as a function of $B$. Thus, the smaller $B$ (the larger the specific solid-fluid heat transfer $\kappa_{s f}$ ), the higher the 'instantaneous damping' of the cooled fluid front by the solid phase. On the other hand, the duration of the first stage is independent of $B$, and it is about equal to the 
characteristic time for a particle of fluid to flow the length $Z_{R}$ at the steady velocity $\mathrm{v}^{\infty}$,

$$
t_{c}^{\text {stage } 1}=\frac{Z_{R}}{\mathrm{v}^{\infty}} \approx 2 \times \tau_{S 1}=13 \text { days } .
$$

(2) During the second stage, the dimensionless temperature remains constant and displays a plateau. Heat transfer between the solid and the fluid phases takes place partly at constant fluid temperature.

(3) The third stage is characterized by a second strong increase of the temperature. The time required for the outlet temperature $T_{f \text {, out }}$ to reach $95 \%$ of the injection temperature $T_{\text {inj }}$ is higher for a model in LTNE than in LTE. In other words, the response of the mixture is delayed by the transfer of heat from the solid phase to the fluid phase. This type of behavior is characteristic of the response of reactive flows and of phases in non-equilibrium: as an example, the consolidation time of a dual porosity medium is delayed compared with a single porosity medium [40].

\subsection{Influence of the fracture porosity $n_{f}$}

The influence of the fracture porosity, in the range of $0.001<n_{f}<0.01$, is mainly visible during the first and the second stage of the double-step pattern (Fig. 3).

(1) For each fracture spacing $B$, the magnitude of $T_{D}$ at the end of this first stage is increasing as a function of the fluid volume ratio $n_{f}$, i.e. the larger the volume of cooled fluid, the smaller the temperature outlet. Since $n_{s} \approx 1$, the rate of heat transfer can be considered as constant, eqn (42), as opposed to the overall amount of heat supply in the system. In addition, the duration of the first stage is independent of $n_{f}$ in agreement with eqn (48).

(2) Time profiles in Fig. 3 indicate that increasing the porosity of the fracture network $n_{f}$ reduces the time length of the second stage and, hence, speeds up thermal depletion. An increase of fluid porosity modifies the phase and the heat partition in the system and hence reduces the overall amount of heat to be transferred by the solid to the fluid. Since the rate of heat transfer is almost independent of the fluid porosity, see eqn (42) in which $n_{s} \approx 1$, the heat transfer period requires less time.

In terms of fracture spacing, the threshold between LTE and LTNE decreases with increasing fluid porosity, $B_{T}=6 \mathrm{~m}\left(n_{f}=0.001\right), B_{T}=2.5 \mathrm{~m}\left(n_{f}=0.005\right)$ and $B_{T}=2 \mathrm{~m}\left(n_{f}=0.01\right)$, due to the increasing amount of cooled fluid. Those values are obtained by trial and error and correspond to $T_{s}=T_{f}$, at the reservoir outlet, over the entire circulation test. For smaller fracture spacings $B<B_{T}$, the local thermal non-equilibrium responses are indistinguishable from the responses in equilibrium and display overshooting oscillations due to an imperfect damping of the convective contribution, see Remark 5.1. The corresponding dimensionless values of $\eta_{D}$ are equal respectively to $11\left(n_{f}=0.001\right), 13\left(n_{f}=0.005\right)$ and $10\left(n_{f}=0.01\right)$. Within the range of interest $0.001 \leq n_{f} \leq 0.01$, dimensionless values $\eta_{D} \geq 13$ are likely to represent LTE and $\eta_{D}<13$ represents LTNE.

The response of a reservoir endowed with a porosity $n_{f}=0.005$ is scrutinized next (Sect. 5.3) for several pumping rates. To maintain LTNE characterized by $\eta_{D}=13$, we expect the fracture spacing threshold $B_{T}=2.5 \mathrm{~m}$ to decrease for larger pumping rates, in view of eqn (47).

Remark 5.1. Results corresponding to $B=1 \mathrm{~m}$ in Fig. 3 are obtained with a model in LTNE and display high overshootings at large times if a standard Galerkin approach is used. These overshootings are associated with the large specific solid-fluid heat transfer coefficient $\kappa_{\text {sf }}$ which plays the role of a penalty coefficient in eqn (13), and induces the development of local sharp temperature gradients. These numerical wiggles are damped by the SUPG and Discontinuity Capturing (DC) methods albeit not perfectly. The simulations have also been performed with a mixture in thermal equilibrium, namely a single temperature. The curves superpose, to within the fact that the single temperature simulations do not show overshootings.

\subsection{Influence of the pumping rate}

For the forced convection phenomenon to be dominant over conduction and for the economical viability of a given reservoir, the fluid velocity in the fracture network should be in the range of $1.0 \times 10^{-7} \mathrm{~m} / \mathrm{s}<$ 

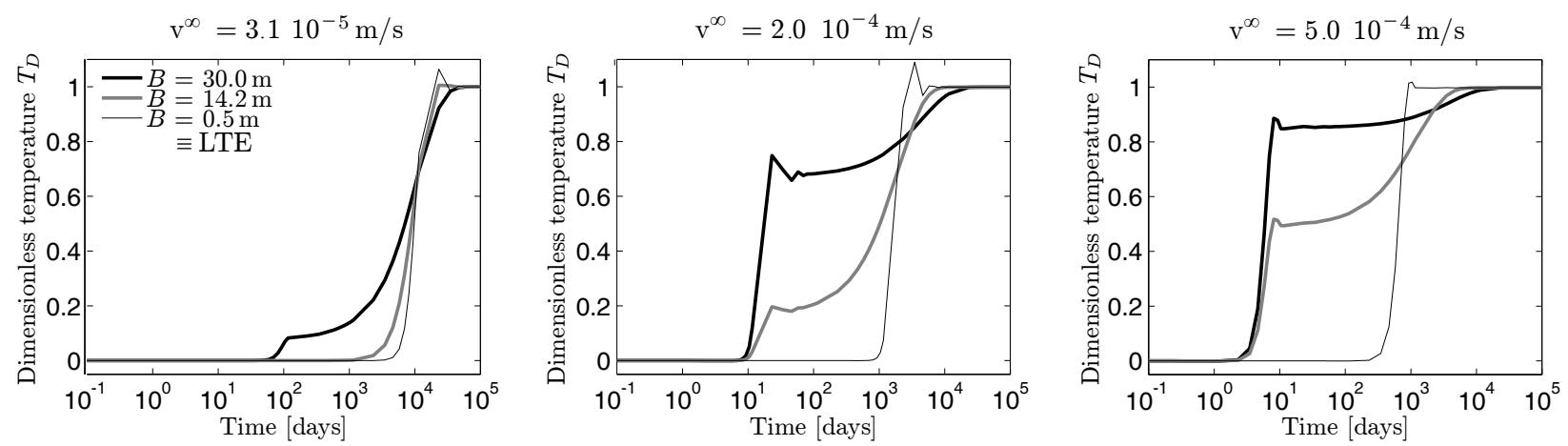

Figure 4: Dimensionless temperature outlet $T_{D}$ as a function of time, for a porosity $n_{f}=0.005$, three steady state fluid velocities $\mathrm{v}^{\infty}$ and three fracture spacings $B$. All results are for $Z_{R}=230 \mathrm{~m}$ and thermal properties from Table 2 . In LTNE, time profiles display a double-step pattern, whereas, in LTE $(B=0.5 \mathrm{~m})$, time profiles display a continuous pattern. The thresholds between LTE and LTNE express in terms of fracture spacing $B_{T}=7.5 \mathrm{~m}(\mathrm{left}), B_{T}=2.5 \mathrm{~m}(\mathrm{middle}), B_{T}=1.5 \mathrm{~m}$ (right). The resulting dimensionless threshold $\eta_{D} \simeq 13$ applies to all velocities.

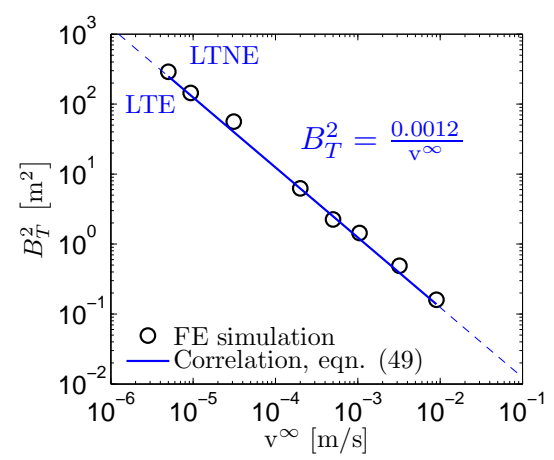

Figure 5: Square of the fracture spacing threshold $B_{T}^{2}$ as a function of the average fluid velocity at steady state ${ }^{\infty}$, for a porosity $n_{f}=0.005$, a uniform flow path and an insulated reservoir. All results are for $Z_{R}=230 \mathrm{~m}$ and thermal properties from Table 2. The hyperbolic relationship defined by eqn (49) is represented by a blue line. Colors are available on the electronic version. It is well captured by the finite element (FE) simulation.

$\mathrm{v}^{\infty}<1.0 \times 10^{-3} \mathrm{~m} / \mathrm{s}[3,5,6]$. Within this range of fluid velocity and for a fracture spacing from 0.5 to $30 \mathrm{~m}$ (so as to capture the LTE threshold for all velocities, see Sect. 5.2), the relevance of the LTNE hypothesis is scrutinized by using the double-step pattern as an indicator.

Results for time profiles with a constant fracture porosity $n_{f}=0.005$ are reported in Fig. 4 for three fluid velocities $\mathrm{v}^{\infty}$ and three fracture spacings $B$.

For an average fluid velocity $\mathrm{v}^{\infty}=2.0 \times 10^{-4} \mathrm{~m} / \mathrm{s}$, the fracture spacing thresholds between LTNE and LTE remains $B_{T}=2.5 \mathrm{~m}$. As expected, this threshold is reduced for larger fluid velocities, for e.g. $B_{T}=1.5 \mathrm{~m}$ for $\mathrm{v}^{\infty}=5.0 \times 10^{-4} \mathrm{~m} / \mathrm{s}$ and is increased for smaller fluid velocities, for e.g. $B_{T}=7.5 \mathrm{~m}$ for v ${ }^{\infty}=3.1 \times 10^{-5} \mathrm{~m} / \mathrm{s}$. Recall that spacing $B$ larger than $B_{T}$ implies LTNE. Below the threshold values, the rate of thermal convection-diffusion in the fractures is comparable to the thermal diffusion in the rock matrix and hence a local thermal non-equilibrium analysis is not required. Consistently, the double-step pattern, in which heat transfer between the solid phase and the fluid phase is characterized by a plateau, is observed from approximatively twice those values. The double-step pattern does not provide an accurate tool to identify the onset of LTNE and is rather a convenient visual control of established LTNE.

As expected, the above thresholds result from eqn (47),

$$
\left(B_{T}\right)^{2}=\frac{1}{\eta_{D}} \frac{1}{n_{f}} \frac{16 \Lambda_{f}}{\Lambda_{s}+2 \Lambda_{f}} \frac{Z_{R} \alpha_{T, f}}{\mathrm{v}^{\infty}},
$$



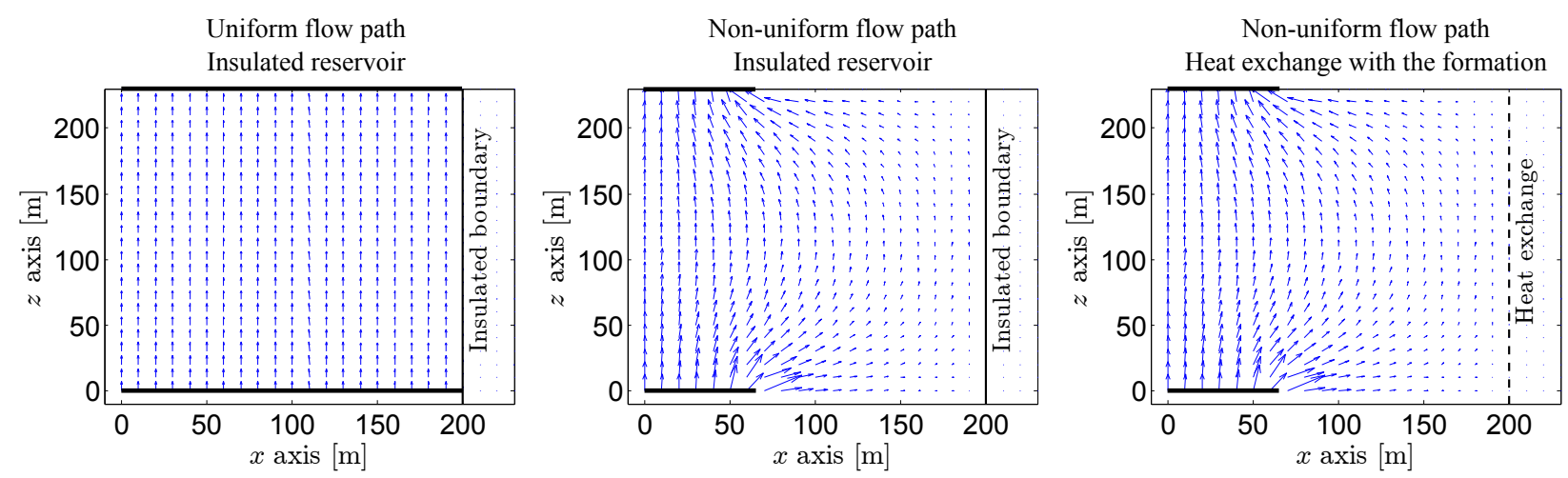

Figure 6: Scaled fluid velocity vectors in the reservoir. Wells (thick horizontal lines) penetrate the reservoir either totally (left) or partially (center, right). The reservoir is insulated from the rock formation (left, center) or exchanges heat with the formation (right).

in which the dimensionless LTNE parameter $\eta_{D}$ takes the value identified in Sect. 5.2 for $n_{f}=0.005$, i.e. $\eta_{D}=13$. A good agreement for the square of the fracture spacing threshold $\left(B_{T}\right)^{2}$ as a function of the fluid velocity $\mathrm{v}^{\infty}$ is obtained between the numerical response and the above correlation, as illustrated in Fig. 5 .

\subsection{Influence of the flow path}

Results reported so far correspond to a uniform vertical flow (Fig.6, left). The influence of a nonuniform flow path on the fracture spacing threshold $B_{T}$ for $n_{f}=0.005$ is addressed for wells shorter than the horizontal extent of the reservoir (Fig. 6, center).

The limit between a single-step pattern and a double-step pattern is lowered to $B_{T}=16 \mathrm{~m}\left(\mathrm{v}^{\infty}=\right.$ $2.0 \times 10^{-4} \mathrm{~m} / \mathrm{s}$ ) when the flow field is non-uniform. The reason is attributed to the fluid velocity in the $x$-direction. For a non-uniform flow distribution, the fluid velocity in the $x$-direction is not negligible and the heat transport is convection-dominated in the $x$ - and $y$-directions. This change in heat transport regime in the $x$-direction increases the rate of the thermal depletion of the solid phase and requires a smaller rate of heat transfer to maintain LTNE. The hyperbolic relation eqn (49) between the square of the fracture spacing threshold and the fluid velocity becomes $\left(B_{T}\right)^{2}=0.051 / \mathrm{v}^{\infty}$, which remains in very good agreement with the numerical data (not shown).

Overall, the comparison between a uniform and a non-uniform flow field yields the conclusion that the flow path (aside from the reservoir volume) has a marked influence on the dimensionless LTNE threshold. For a given reservoir (e.g. $B_{T}=2.5 \mathrm{~m}$ is obtained from microseismic monitoring techniques), the above correlation provides valuable information on the minimum fluid velocity which should be $\mathrm{v}^{\infty}=2.0 \times 10^{-4} \mathrm{~m} / \mathrm{s}$ for a uniform flow field (the fracture network is fully penetrated by the wells) and $\mathrm{v}^{\infty}=8.0 \times 10^{-3} \mathrm{~m} / \mathrm{s}$ for a non-uniform flow field (the fracture network is larger than the wells).

\subsection{Influence of the external heat supply}

Heat recovered from a geothermal reservoir is captured a priori from the reservoir itself. However, the rock formation in contact with the reservoir may also contribute, depending on the thermal boundary condition. Two extreme situations are compared: (1) the reservoir is insulated from the rock formation boundary along the boundary $x=X_{R}$ (Fig. 6, center); (2) the reservoir is in direct contact with the low permeability rock formation $\left(k_{f}=10^{-20} \mathrm{~m}^{2}\right)$ which is continuously heated by the surrounding earth, $T_{s}=T_{f}=T^{0}$, along its boundary $x=X_{F}$ (Fig. 6, right). In this second case, the results are strongly influenced by the formation width $X_{F}-X_{R}$. A conductive boundary is recovered as this width is increased to infinity. No experimental data are available to calibrate the width of the rock formation that thermally contributes to the reservoir (Sect. 6). The arbitrary value of $X_{F}-X_{R}=30 \mathrm{~m}$ is discussed below.

For $n_{f}=0.005$ and non-uniform flow of $\mathrm{v}^{\infty}=2.0 \times 10^{-4} \mathrm{~m} / \mathrm{s}$, the limit between a single-step pattern and a double-step pattern corresponds to $B_{T}=3 \mathrm{~m}$. The contribution of the rock formation reduces the rate of 


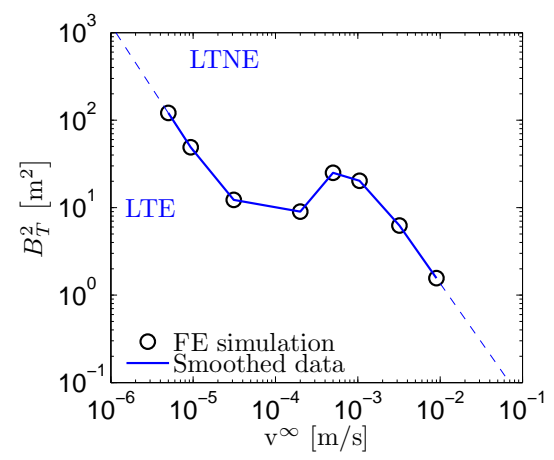

Figure 7: Same as Fig. 5 but for a non-uniform flow path and heat exchange with a formation of $30 \mathrm{~m}$ width. The drawdown results correspond to the tip of the production well, i.e. $x=X_{W}=60 \mathrm{~m}$. A non-linear non-monotonic response is obtained from the finite element (FE) simulations in opposition with the power response suggested by eqn (49). Heat exchange between the reservoir and the rock formation requires the use of the SUPG method, Remark 5.3.

the thermal depletion of the fluid which may never reach $T_{D}=0.95$, for small flow rates. The additional heat provided by the formation in the neighborhood of the production well is little affected by convection due to small fluid velocities in this area (Fig. 8, right). The relation between the square of the fracture spacing threshold and the inverse of the flow rate is no longer linear and even non-monotonic (Fig. 7). The non-monotonic behavior can be further explained by looking at the characteristic times. At high fluid velocities $\mathrm{v}^{\infty}>10^{-3} \mathrm{~m} / \mathrm{s}$, the time required for the thermal depletion of the reservoir is much smaller than the characteristic time required by diffusion in the hot formation. The hot formation is merely equivalent to a zero heat flux boundary condition along the line $x=X_{R}$, for the time span over interest.

For intermediate flow rates, the characteristic times of diffusion in the rock formation and of thermal depletion in the reservoir are of similar magnitude. The externally supplied heat is mainly transported across the reservoir by the convective fluid so that the depletion time of the fluid phase is increased. Although the fluid velocity is increased, the fluid temperature remains high due to the external heat supply. Hence, LTNE is only important if the fracture spacing is large.

Remark 5.2. Instead of including a rock formation in the finite element mesh, the influence of the hot formation can be introduced via a convective boundary condition,

$$
\mathbf{q}_{k} \cdot \hat{\mathbf{n}}=h_{k, \text { earth }}\left(T_{k}-T_{\text {earth }}\right), \quad k=s, f,
$$

in which $h_{k, \text { earth }}$ is the heat transfer coefficient $\left[W / m^{2} . K\right]$ between the phase $k$ and earth, and $T_{\text {earth }}$ is the constant temperature of the earth. The heat transfer coefficients are defined by the thermal conductivities weighted by a coefficient $W: h_{f, \text { earth }}=W n_{f} \Lambda_{f} /\left(X_{F}-X_{R}\right), h_{s, \text { earth }}=W n_{s} \Lambda_{s} /\left(X_{F}-X_{R}\right)$ and $T_{\text {earth }}=T^{0}$. The case of a reservoir surrounded by a formation of width $X_{F}-X_{R}=30 \mathrm{~m}$ is recovered for a precise value of $W$. For example, if the injection rate is $\mathrm{v}^{\infty}=2.0 \times 10^{-4} \mathrm{~m} / \mathrm{s}$, the weighting coefficient $W$ is equal to 0.17 so as to recover the same thermal depletion curve. The convective boundary condition is particularly appropriate for industrial heat exchangers where the cooling/heating temperature $T_{\text {earth }}$ is enforced at a known distance with a cooling/heating loop.

Remark 5.3. Heat exchange between the reservoir and the surrounding rock formation, by direct contact or via a convective boundary condition, requires the use of the SUPG method [27, 29]. Indeed, the boundary condition induces a sharp temperature gradient, which may lead to spurious numerical wiggles if the numerical response is not stabilized appropriately. The response for the Galerkin formulation is highly oscillatory, Fig. 8. However efficient, the SUPG method is not perfect [41] and significant overshootings remain along the boundary. These overshootings may be damped by use of the discontinuity capturing method, Fig. D.15. Still, the discontinuity capturing method has been experienced to be prone to instabilities for large velocities and large times. 
With the Galerkin method

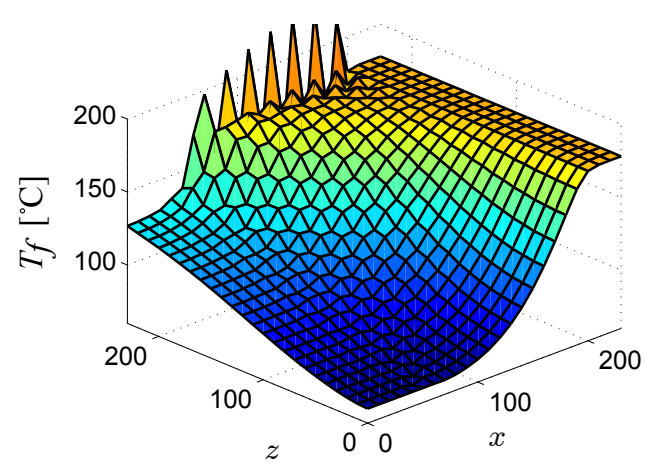

With the SUPG method

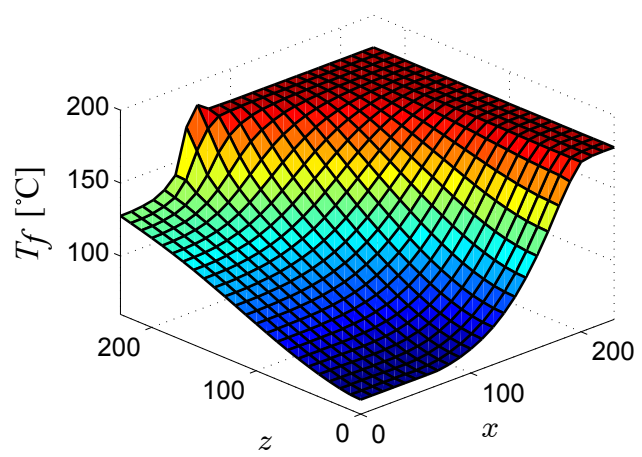

Figure 8: Fluid temperature contours for $\mathrm{v}^{\infty}=2.0 \times 10^{-4} \mathrm{~m} / \mathrm{s}$, at $t=3.18$ years, accounting for heat transfer with the rock formation and for a non-uniform flow field. The Galerkin method (left) displays spurious numerical wiggles, which are partly cured by the SUPG method (right).

\begin{tabular}{lccc}
\hline \hline Initial or boundary conditions & Value & Unit & Reference \\
\hline Injection temperature $T_{f, \text { inj }}$ & 70 & ${ }^{\circ} \mathrm{C}$ & {$[42]$} \\
Initial in-situ temperature $T^{0}$ & 178 & ${ }^{\circ} \mathrm{C}$ & {$[42]$} \\
Initial production pressure $p_{f, \text { out }}^{0}=\rho_{f} g H$ at $H=2.673 \mathrm{~km}$ & 26.19 & $\mathrm{MPa}$ & Sect. 4.3 \\
Initial injection pressure $p_{f, \text { inj }}^{0}=\rho_{f} g H$ at $H=2.903 \mathrm{~km}$ & 28.44 & $\mathrm{MPa}$ & Sect. 4.3 \\
Injection overpressure & 9.0 & $\mathrm{MPa}$ & {$[42]$} \\
Overburden compressive stress & -75.0 & $\mathrm{MPa}$ & {$[43]$} \\
Lateral earth stress & -37.5 & $\mathrm{MPa}$ & {$[43]$} \\
\hline \hline
\end{tabular}

Table 1: Initial and loading boundary conditions representative of Fenton Hill HDR reservoir, run segment 5.

\section{Fenton Hill HDR reservoir}

The thermal responses elicited from the thermo-hydro-mechanical model in LTNE may be back-calculated with results from a 300-day circulation test at the Fenton Hill HDR reservoir, New Mexico, USA.

The circulation test was induced between depths $2903 \mathrm{~m}$ and $2667 \mathrm{~m}$ with an average reservoir height $Z_{R}$ of $230 \mathrm{~m} \mathrm{[42].} \mathrm{The} \mathrm{horizontal} \mathrm{half-width} X_{R}$ of the permeable reservoir is arbitrary chosen equal to $200 \mathrm{~m}$ surrounded by a formation of width $X_{F}-X_{R}=30 \mathrm{~m}$. The horizontal half-width of the wells is equal to either (1) $X_{W}=200 \mathrm{~m}$ or $(2) X_{W}=60 \mathrm{~m}$. Loading boundary conditions and material parameters are documented in Tables 1 and 2. The fluid pressures at the injection and the production wells are hydrostatic.

For a typical diffusion length $Z_{R}$ of $230 \mathrm{~m}$ and the material parameters of Table 2, the characteristic times of seepage and of thermal diffusion through the solid skeleton, as defined by eqns (35), are equal to $3500 \mathrm{~s} \simeq 1$ hour and $4.8 \times 10^{10} \mathrm{~s} \simeq 1520$ years, respectively. Therefore, as indicated in Sect. 3.3, the minimum and the maximum time steps are taken equal to $\Delta t_{\min }=1000 \mathrm{~s}$ and $\Delta t_{\max }=10^{10} \mathrm{~s} \simeq 317$ years.

\subsection{Calibration of the model}

The least well-defined of the required material parameters are the fracture permeability $k_{f}$ after hydraulic stimulation, the fracture porosity $n_{f}$ and the specific inter-phase heat transfer coefficient $\kappa_{s f}$.

Experimental data reported by Zyvoloski et al. [42] are used to calibrate the above parameters based on the following procedure: (1) the fracture network permeability $k_{f}$ is obtained so that the first stage of the thermal depletion curve, mainly due to the convection of the fluid, matches the field data; (2) the fracture network porosity $n_{f}$ is adjusted so that the duration of the second stage of thermal depletion matches the rest of the response; finally (3) the optimum inter-phase heat transfer coefficient $\kappa_{s f}$ is obtained by trial and error from the temperature magnitude at the start of the second stage of the field data, Fig. 9. 


\begin{tabular}{lccc}
\hline \hline Material parameter & Value & Unit & Reference \\
\hline Drained Young's modulus $E$ & 38.9 & $\mathrm{GPa}$ & {$[43]$} \\
Drained Poisson's ratio $\nu$ & 0.3 & - & {$[43]$} \\
Bulk thermal expansion coefficient $c_{T}$ & $3.3 \times 10^{-6}$ & $1 / \mathrm{K}$ & {$[42]$} \\
Fissure network porosity $n_{f}$ & $<0.01$ & - & {$[43]$} \\
Fissure network permeability $k_{f}$ & $<1.0 \times 10^{-13}$ & $\mathrm{~m}^{2}$ & {$[43]$} \\
Solid grains compressibility $c_{s}$ & $2.7 \times 10^{-11}$ & $1 / \mathrm{Pa}$ & {$[43]$} \\
Solid thermal conductivity $\Lambda_{s}$ & 2.71 & $\mathrm{~W} / \mathrm{m} . \mathrm{K}$ & {$[5]$} \\
Solid specific heat capacity $C_{s}^{(v)}$ & 948.55 & $\mathrm{~J} / \mathrm{kg} \cdot \mathrm{K}$ & {$[26]$} \\
Solid density $\rho_{s}$ & 2600 & $\mathrm{~kg} / \mathrm{m}^{3}$ & {$[26]$} \\
Solid thermal diffusivity $\alpha_{T, s}$ & $1.1 \times 10^{-6}$ & $\mathrm{~m}^{2} / \mathrm{s}$ & - \\
Fluid hydraulic compressibility $c_{f H}$ & $4.54 \times 10^{-10}$ & $1 / \mathrm{Pa}$ & $\dagger$ \\
Fluid thermal expansion coeff. $c_{f} T$ & $1.0 \times 10^{-3}$ & $1 / \mathrm{K}$ & $\dagger$ \\
Fluid dynamic viscosity $\mu_{f} \ddagger$ & $3.0 \times 10^{-4}$ & $\mathrm{~Pa} . \mathrm{s}$ & $\dagger$ \\
Fluid thermal conductivity $\Lambda_{f}$ & 0.6 & $\mathrm{~W} / \mathrm{m} \cdot \mathrm{K}$ & $\dagger$ \\
Fluid specific heat capacity $C_{f}^{(p)}$ & 4275 & $\mathrm{~J} / \mathrm{kg} \cdot \mathrm{K}$ & {$[5]$} \\
Fluid density $\rho_{f}$ & 980.0 & $\mathrm{~kg} / \mathrm{m}^{3}$ & $\dagger$ \\
Fluid thermal diffusivity $\alpha_{T, f}$ & $1.58 \times 10^{-7}$ & $\mathrm{~m}^{2} / \mathrm{s}$ & - \\
\hline \hline
\end{tabular}

Table 2: Input parameters representative of Fenton Hill HDR reservoir, run segment 5. † Estimated parameters for water and granite. $\ddagger$ Although, fluid dynamic viscosity varies much with temperature [44] and with viscosity-increasing additives or propping agents, the fluid dynamic viscosity $\mu_{f}$ used throughout corresponds to pure water at $95{ }^{\circ} \mathrm{C}$, Table A.2.4 in de Marsily [45].

The calibration is considered for two different reservoir geometries: (i) for $X_{W}=X_{R}$, the flow field is uniform in space, Fig. 9, left; (ii) for $X_{W}<X_{R}$, the flow field is non-uniform in space, Fig. 9, right. In both cases, the reservoir is assumed to exchange heat with the formation. The mechanical boundary conditions are summarized in Sect. 4.4.

If the flow field is uniform, the calibration yields the fracture network permeability $k_{f}=8.0 \times 10^{-15} \mathrm{~m}^{2}$, the porosity $n_{f}=0.005$, and the optimum specific inter-phase heat transfer coefficient $\kappa_{s f}=33 \mathrm{~mW} / \mathrm{m}^{3} . \mathrm{K}$, corresponding to a block width $B=14.2 \mathrm{~m}$ in agreement with the magnitude used in Fig. 3.2 in Zyvoloski et al. [42]. If the flow field is non-uniform, the calibration yields a higher permeability $k_{f}=2.35 \times 10^{-14} \mathrm{~m}^{2}$, the same porosity $n_{f}=0.005$ and a slightly smaller specific inter-phase heat transfer coefficient $\kappa_{s f}=$ $30 \mathrm{~mW} / \mathrm{m}^{3} . \mathrm{K}$.

The inferred fracture network permeability is found to depend strongly on the spatial characteristics of the flow field. As expected, the non-uniform flow field setup requires a higher permeability $k_{f}$, that is a higher average fluid velocity $\mathrm{v}^{\infty}$. Overall, both types of flow fields show a good correspondence with the experimental data and provide the same magnitude for the specific inter-phase heat transfer coefficient $\kappa_{s f} \approx O(10) \mathrm{mW} / \mathrm{m}^{3} . \mathrm{K}$.

Data provided by Zyvoloski et al. [42] display spatially heterogeneous initial temperatures. This situation may be explained by the hydraulic stimulation tests previously undergone by the reservoir. The initial temperature $T^{0}$ used in Fig. 9 represents the initial temperature at $2703 \mathrm{~m}$ depth, i.e. at the lowest point of the production well. If $T^{0}$ is chosen equal to the average temperature of the production well, the results of Fig. 9 hold for higher values of $\kappa_{s f}$. The overall response remains the same but the second stage of thermal depletion begins at a lower dimensionless temperature $T_{D}$.

The initial response (stage 1) is dominated by the immediate response of the convective fluid while stage 2 corresponds to the transfer between the fluid and the solid phase. Only later thermal history ( $>300$ days) is influenced by the external heat supply from the surroundings. Hence, the circulation test is too brief to yield a conclusive comparison on the thermal boundary condition at the reservoir-formation vertical interface, even with a non-uniform flow field. 

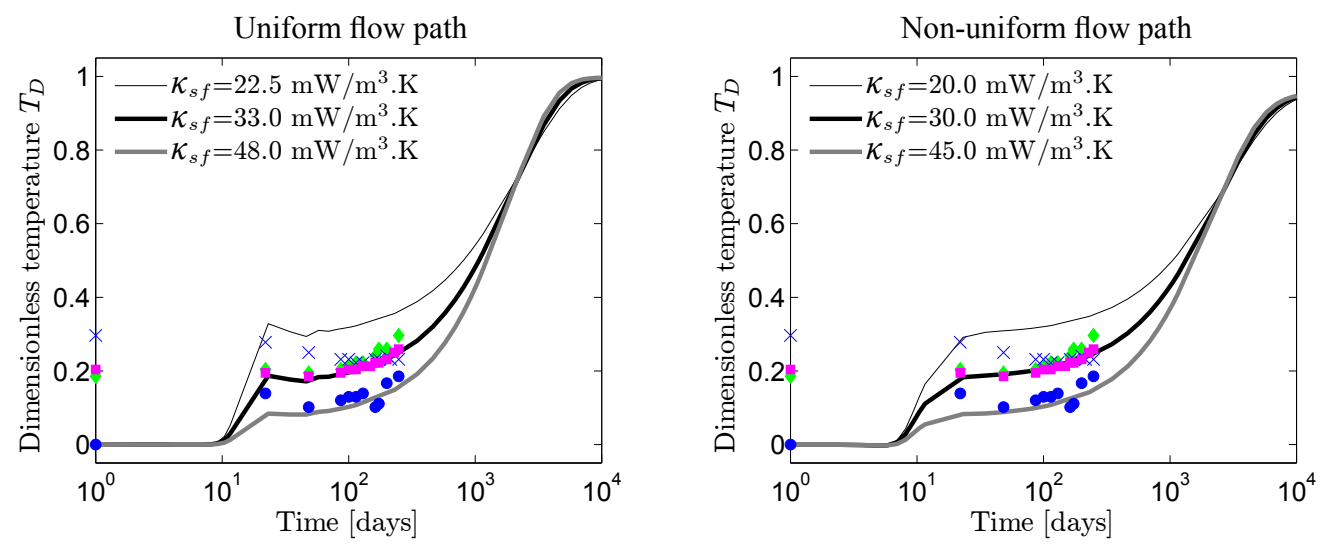

Figure 9: Relative temperature outlet $T_{D}$ versus time $t$ along the production well at $x=60 \mathrm{~m}$. Experimental data pertain to different depths, namely $\circ 2703 \mathrm{~m}, \diamond 2673 \mathrm{~m}, \times 2626 \mathrm{~m}$ and $\square$ in the casing $2660 \mathrm{~m}$. The experimental temperatures at day one result from the spatial heterogeneity along the production well, see text. (left) Uniform flow field, $k_{f}=8.0 \times 10^{-15} \mathrm{~m}^{2}$ and $n_{f}=0.005$. Optimum specific inter-phase heat transfer coefficient $\kappa_{s f}=33.0 \mathrm{~mW} / \mathrm{m}^{3}$.K. (right) Non-uniform flow field, $k_{f}=2.35 \times 10^{-14} \mathrm{~m}^{2}$ and $n_{f}=0.005$. Optimum specific inter-phase heat transfer coefficient $\kappa_{s f}=30.0 \mathrm{~mW} / \mathrm{m}^{3} . \mathrm{K}$.

If, from Fig. 9, left, which is associated with uniform flow, a fracture permeability of $k_{f}=8.0 \times 10^{-15} \mathrm{~m}^{2}$ $\left(\mathrm{v}^{\infty}=2.0 \times 10^{-4} \mathrm{~m} / \mathrm{s}\right)$ is assumed, the entire thermal drawdown history may be determined directly from Fig. 3 for various porosities and heat transfer parameters. Similarly, if a porosity of $n_{f}=0.005$ is assumed for this physical system, the full thermal drawdown history may be determined directly from Fig. 4 for various flow rates and heat transfer parameters.

\subsection{THM coupled behavior}

The LTNE model is used to investigate the coupled thermo-hydro-mechanical behavior of the Fenton Hill HDR reservoir. The material parameters matching the experimental data for a uniform flow field are used, that is $k_{f}=8.0 \times 10^{-15} \mathrm{~m}^{2}, n_{f}=0.005$ and $\kappa_{s f}=33.0 \mathrm{~mW} / \mathrm{m}^{3} . \mathrm{K}$. The reservoir is assumed to exchange heat with the rock formation, although the results are not affected by the external heat supply for the specific case considered. The mechanical boundary conditions prior to the circulation test are presented in Sect. 4.3 and are maintained constant throughout the simulations.

The coupled behavior of porous media in LTNE is governed by the difference in characteristic times between the thermal depletion of the fluid phase and that of the solid phase, Fig. 10. For example, at roughly 5.8 days the thermal depletion of the fluid phase has already started to propagate inside the reservoir, whereas the thermal depletion of the solid phase is confined to the immediate vicinity of the injection well. However, as heat transfer between the two constituents gets completed, the mixture tends to thermal equilibrium.

This early significant difference in time depletion rates is due to the volume difference between the solid skeleton and the fluid. Indeed, the fluid porosity is $n_{f}=0.005$, and the fracture aperture is $2 b=0.035 \mathrm{~m}$ for a typical fracture spacing of $B=14.2 \mathrm{~m}$. Hence, although local thermal equilibrium is 'almost' instantaneous along the fracture walls, the width of the solid blocks (perpendicular to the fractures) is much larger than the aperture of the fractures; and therefore at the scale of the solid blocks the time required to cool down the solid phase (by diffusion of heat from the solid block) is much larger than that for the fluid phase.

In transient convection-dominated diffusion convection problems, two types of numerical noises are encountered: (1) the first type is observed during the initial times due to the application of a sharp temperature gradient at the injection well which, analogous to a shock front, disappears after a few time steps $[46,47]$; (2) the second type is observed later when the thermal front hits a prescribed boundary condition. The SUPG method was initially designed to cure the second type of numerical noise for steady state convection-dominated diffusion convection problems [27]. However, if the SUPG method is used for transient convection-dominated diffusion convection problems, the magnitude of the early time noise is enlarged compared with the Galerkin approximation. This problem is tackled using the method proposed by Tezduyar 

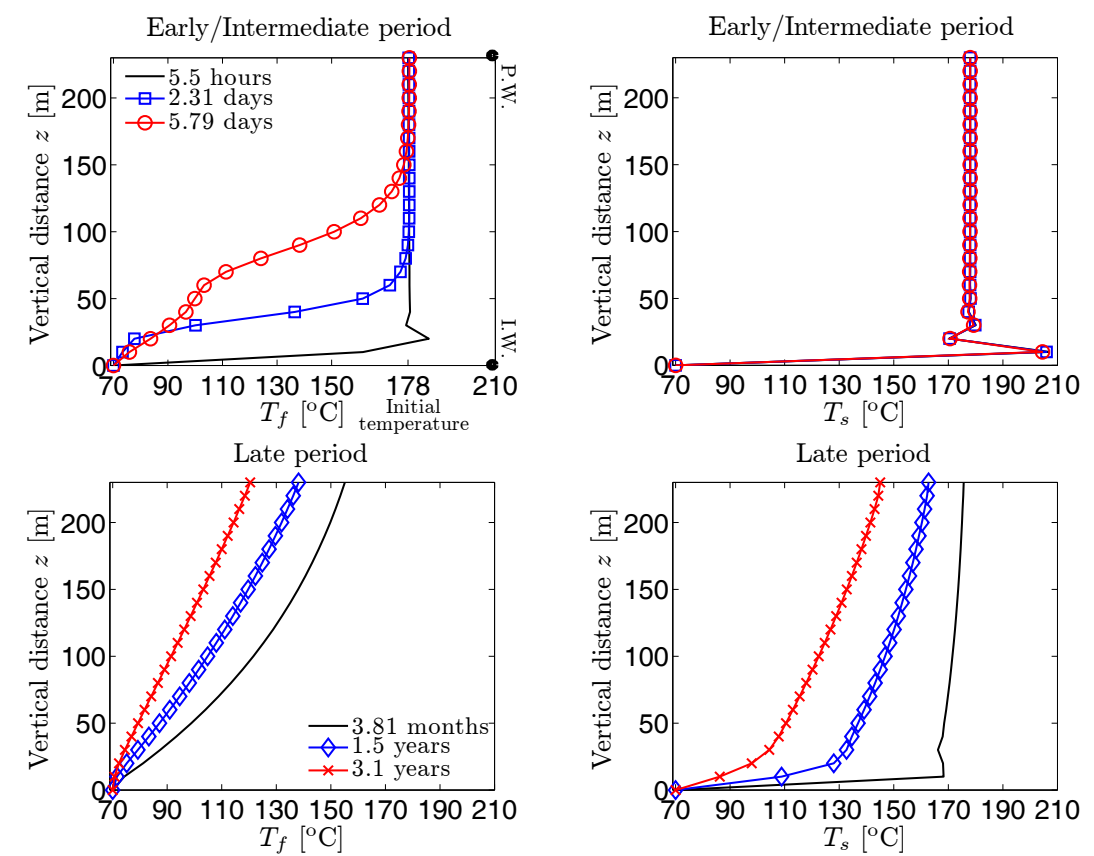

Figure 10: Profiles, along the $z$-axis at $x=60 \mathrm{~m}$, of fluid temperature (left) and solid temperature (right), with $n_{f}=0.005$, $k_{f}=8.0 \times 10^{-15} \mathrm{~m}^{2}, \kappa_{s f}=33.0 \mathrm{~mW} / \mathrm{m}^{3} . \mathrm{K}$ and a uniform flow field. P.W. stands for production well and I.W. for injection well. The early wiggles near the injection well are numerical artifacts due to an imperfect damping of the convective contribution, see text. The thermal depletion of the fluid phase is significantly ahead of the thermal depletion of the solid phase.
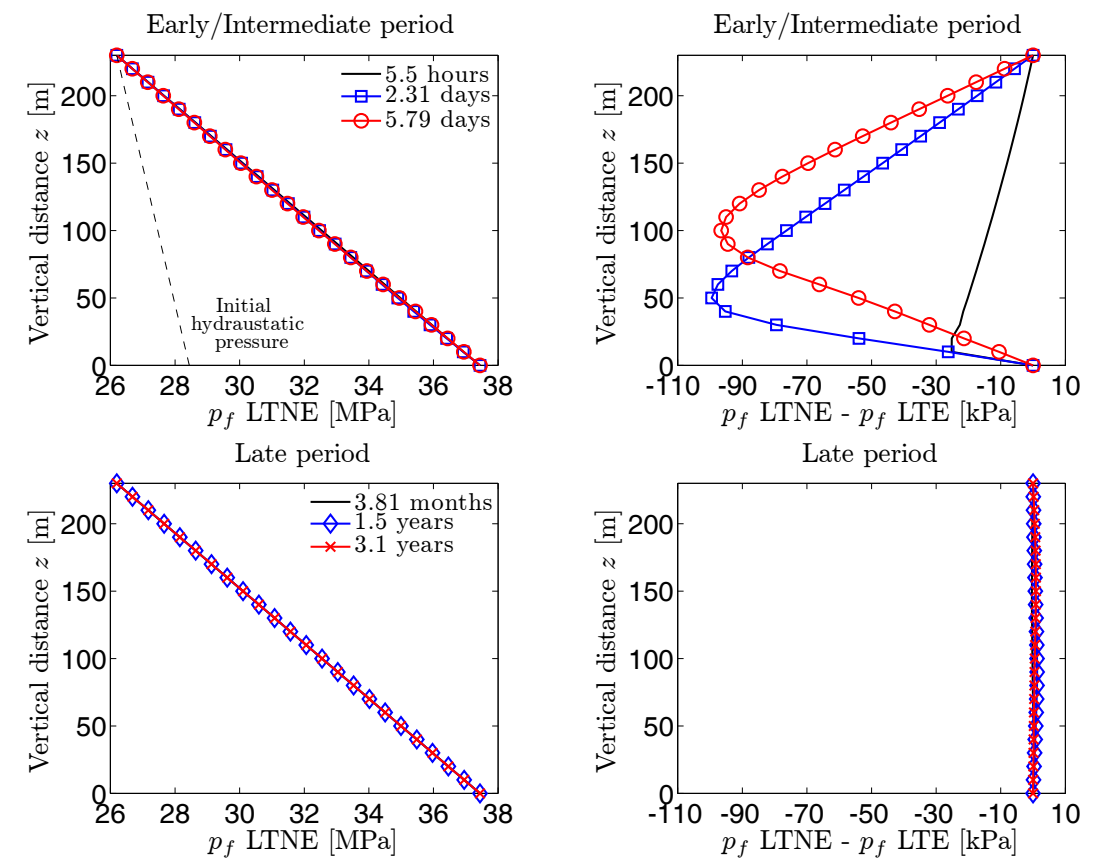

Figure 11: Profiles, along the $z$-axis at $x=60 \mathrm{~m}$, of fluid pressure $p_{f}$ (left) and relative fluid pressure with respect to the LTE response (right). Same parameters as Fig. 10. The fluid pressure reaches quickly steady state and remains undisturbed in spite of the thermal depletion of the fluid phase and of the thermal contraction of the solid phase. 

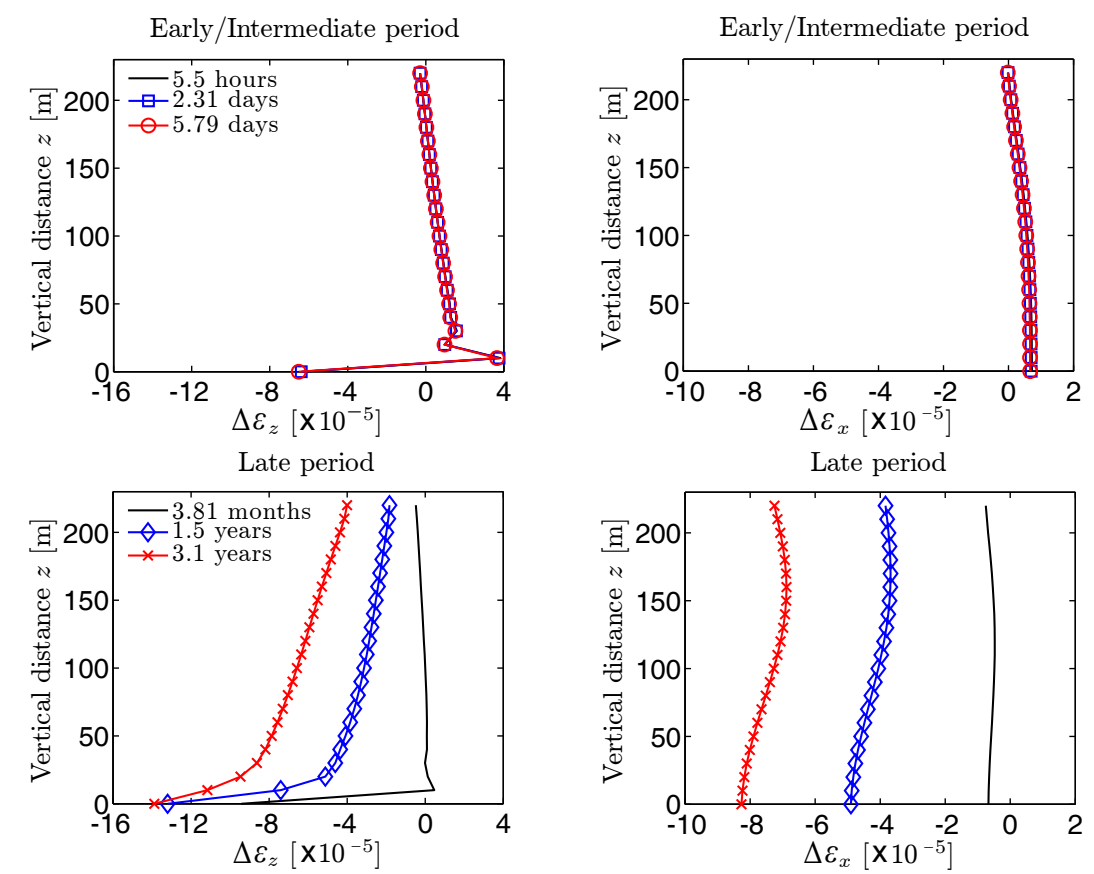

Figure 12: Profiles, along the $z$-axis at $x=60 \mathrm{~m}$, of vertical strain (left) and lateral strain (right). Same parameters as Fig. 10. Extension is counted positive. The simulations assume a plane strain analysis, $\epsilon_{y}=0$. Contraction of the solid phase, $\Delta \epsilon_{z}<0$ and $\Delta \epsilon_{x}<0$, is controlled by the solid temperature response and, thus, develops in the late period.

and Osawa [29], eqn (18): the magnitude of the first kind of numerical noise is decreased with respect to a Galerkin approximation and the second type of noise is efficiently cured.

This issue is particularly important in coupled problems since the early numerical noise in the fluid temperature may pollute the response of the other fields, see Figs. 10 to 13 during the early/intermediate period.

The pressure field reaches steady state within 1 hour due to the high hydraulic diffusivity of the fracture network, Fig. 11, left. To highlight the influence of the LTNE assumption, the pressure responses in LTNE $\left(\kappa_{s f}=33 \mathrm{~W} / \mathrm{m}^{3} . \mathrm{K}\right)$ and in LTE $\left(\kappa_{s f}=100 \mathrm{~W} / \mathrm{m}^{3} . \mathrm{K}\right)$ are compared in Fig. 11 . The very little difference (during the early/intermediate period) is associated with distinct rates of thermal depletion of the fluid phase. The pressure drops at $t=2.31$ and 5.79 days are induced by the fact that the fluid phase is embedded in a more rigid solid skeleton, thermally undisturbed at short times. Indeed, the drained thermal expansion/contraction of the mixture is controlled locally by the solid phase and is independent of the change in fluid temperature, until energy exchange between the phases takes place, giving rise to a solid temperature variation. However, these thermally induced pressure drops remain very small in magnitude, i.e. a few $\mathrm{kPa}$, due to the large permeability of the fracture network.

The strain response is associated with the thermal depletion of the solid phase, Fig. 12. The latter takes place during the late period and induces a contraction of the solid skeleton in both lateral and vertical directions. The contractive strains increase over time but remain largest near the injection well where cooling takes place first. Consequently, the strains remain small during the early/intermediate periods. The slight noise due to the overshooting of the temperature of the solid phase has limited impact. Note that the absence of noise in the lateral strain profile is due to a smooth temperature gradient in the $x$-direction.

The effective stresses are similarly governed by the thermal depletion of the solid phase during the late period, Fig. 13. Cooling of the lower part of the reservoir induces contractive strains and tensile stresses. A sort of arching develops in the upper part of the reservoir where a compressive effective horizontal stress develops in time. The limit response of the reservoir is reached at about 30 years, as might be guessed from the stress profile at a later time displayed on Fig. 14. 

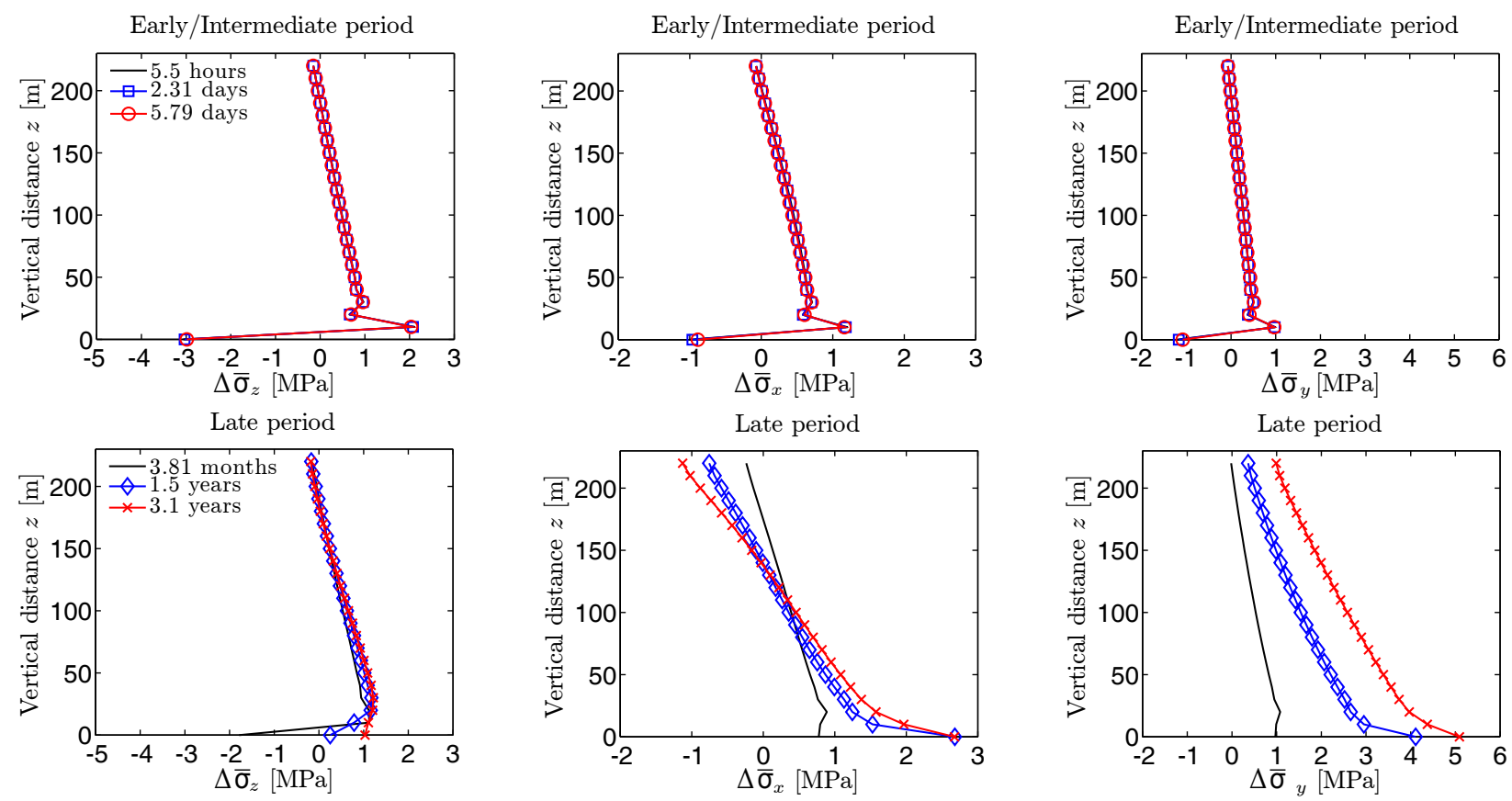

Figure 13: Profiles, along the $z$-axis at $x=60 \mathrm{~m}$, of the induced change in vertical effective stress (left), lateral effective stress (center) and out of plane effective stress (right). Same parameters as Fig. 10. Tensile stresses are counted positive. The contraction of the solid phase induces the effective lateral stress to be tensile near the injection well $(z<120 \mathrm{~m})$ and compressive near the production well $(z>120 \mathrm{~m})$.

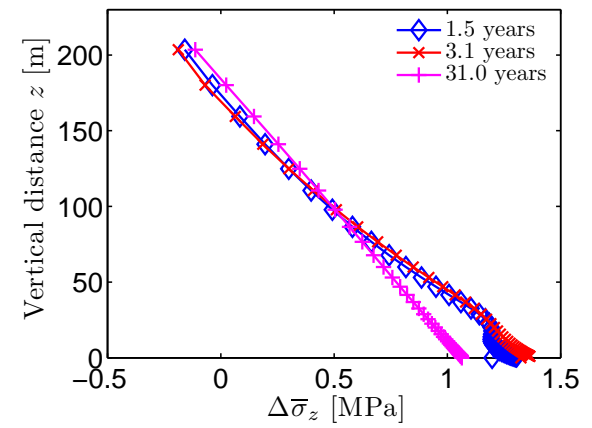

Figure 14: Same as Fig. 13 but with a refined mesh in the vicinity of the injection well. The profile at 31.0 years corresponds to the complete cooling of the reservoir.

The large difference between the characteristic times of the fracture fluid response (early/intermediate period) and of the solid temperature response (late period) allows segregation of the respective contributions to the effective stress due to hydraulic and thermal effects. As expected, the latter is larger than the former especially in the horizontal and out of plane directions.

Tensile stresses induced by the contraction of the rock may cause the aperture of the fractures to increase or/and could initiate new vertical fractures in the $x-z$ and $y-z$ planes from the solid phase. Conversely, compressive stresses close to the injection well (early period) and near the production well (late period) could trigger the creation of shear bands. None of these coupled effects are accounted for in this study. The potential increase in permeability of the voids (or micro-fractures) perpendicular to the main flow path may lead to an increase of fluid loss. Conversely, an increase of fracture aperture in the $x-z$ plane would favor the 'growth' of the reservoir. 
It is worth noting that a variation of aperture could significantly influence the fluid distribution and the rate of thermal depletion. If the injection is controlled by a constant pressure gradient, a variation of aperture would modify the convective velocity through the balance of mass of the fluid. Hence, the above predictions of heat extraction are valid if the thermally stress-induced aperture change is negligible. If the injection is controlled by a constant flow rate, a variation of aperture would modify the fluid pressure [48] and perhaps trigger further thermo-mechanical couplings that deserve to be addressed.

\section{Conclusions}

Diffusion and forced convection mechanisms between two phases have been studied in the context of heat extraction from a fractured hot dry rock reservoir. A model describing the behavior of poroelastic fractured media has been extended to account for local thermal non-equilibrium. The fully coupled thermo-hydromechanical system has been specified for single porosity mixtures. The nonlinear field equations associated with a Galerkin finite element discretization have been stabilized with a Petrov-Galerkin method and solved using a full Newton-Raphson procedure. This finite element analysis has been employed to investigate the response of a generic hot dry rock reservoir.

In the current analysis, no attempt has been made to capture the growth of the fracture network due to the thermal recovery process. Evolution of crack length and aperture due to thermo-mechanical loadings will be the subject of a subsequent study.

A parametric analysis has been carried out to study the influences of the solid-fluid heat transfer coefficient, the fluid porosity and the flow rate. As a typical feature of local thermal non-equilibrium, the temperature outlet profile displays a double-step pattern, representing the three-stage response of the reservoir, namely 1 . the initial convection of the fluid; 2 . the transfer of heat between the solid and the fluid, and 3. the final depletion of the mixture. The solid-fluid heat transfer coefficient controls the characteristic time to recover local thermal equilibrium. Porosity influences the duration of stage 2. Large flow rates favor the time difference between heat propagation in the fluid and in the solid, and therefore the double-step pattern. The influences of the spatial uniformity of the flow path and of the external heat supply on the thermal depletion of the reservoir have also been scrutinized. The performance of the system appears very sensitive to the spatial distribution of the flow path and less sensitive to the amount of external heat supply.

The model is used to describe the thermo-hydro-mechanical behavior of a long term circulation test on the Fenton Hill HDR reservoir. The least well-defined material parameters have been calibrated with experimental data and the comparison with the numerical results demonstrates a local thermal non-equilibrium response. It has been found that the thermally induced fluid pressure drop is very small in magnitude and is confined to the early time response. Later, the thermally induced effective stresses are tensile near the injection well due to the thermal contraction of the solid, suggesting a possible increase of fracture aperture, and compressive near the production well due to the pull-in of the producing area.

This work highlights how LTNE versus LTE can strongly impact the mechanical response of the reservoir and possibly affect the evolution of the fracture network. The LTNE model leads to a characteristic doublestep reservoir response. This feature is clearly identified in the global performance of the Fenton Hill reservoir. In particular, the double-step history of the outlet temperature shown on Figure 9 could not be recovered by a single temperature model (standard poroelasticity).

Here, the solid blocks are endowed with their own temperature, but they are impermeable to fluid. A more general model would account for permeable porous blocks, and thus introduce the temperatures of the solid, the temperature of the fluid of the pores and the temperature of the fluid of the fracture, as well as the pressures associated with these two fluids. Still, in a geothermal context, the seepage of fluid through the porous blocks may be considered so slow, that the temperatures of the fluid of the pores and the solid are equal. Consequently, the improved model would be content to introduce the pressure of the pores as an additional unknown. The influence of a second porosity and the mechanisms of fluid loss are addressed in a separate paper [39]. The improved model introduces the pressure of the pores as an additional unknown and shows that the seepage of the fluid through the pores reduces the tensile stress. 


\section{Notation}

Roman letters

$A$ Skempton coefficient, eqn (31) [-]

$b$ half width of a fracture $[\mathrm{m}]$

$B$ width of the porous blocks $[\mathrm{m}]$

$c_{\mathrm{DS}}$ drained compressibility of solid skeleton $[1 / \mathrm{Pa}]$

$c_{f H}$ compressibility of the fluid $[1 / \mathrm{Pa}]$

$c_{s}$ compressibility of the solid grains $[1 / \mathrm{Pa}]$

$c_{T}$ coefficient of thermal expansion of the mixture $[1 / \mathrm{K}]$

$c_{k T}$ coefficient of thermal expansion of phase $k[1 / \mathrm{K}]$

$C_{k}^{(p)}$, resp. $C_{k}^{(v)}$, specific heat capacity

at constant pressure, resp. volume, of phase $k[\mathrm{~J} / \mathrm{kg} . \mathrm{K}]$

$E$ drained Young's modulus [Pa]

$H_{k}$ enthalpy of phase $k[\mathrm{~J} / \mathrm{kg}]$

$h, h_{\|}$element length $[\mathrm{m}]$

$h_{s f}$ solid-fluid heat transfer coefficient $\left[\mathrm{W} / \mathrm{m}^{2} . \mathrm{K}\right]$

$\mathbf{j}_{f}$ fluid flux in the fractures $[\mathrm{m} / \mathrm{s}]$

$k_{f}$ fracture permeability $\left[\mathrm{m}^{2}\right]$

$\mathbf{N}$ interpolation function [-]

nel number of elements [-]

neq number of equations [-]

$n_{k}$ porosity of phase $k\left[\mathrm{~kg} / \mathrm{m}^{3}\right]$

Pe Péclet number, eqn (46) [-]

$\mathrm{Pe}_{\mathrm{g}}$ grid Péclet number [-]

$p_{f}$ pressure of the fluid $[\mathrm{Pa}]$

$\mathbf{q}_{k}$ heat flux in phase $k\left[\mathrm{~W} / \mathrm{m}^{2}\right]$

$s_{k}$ apparent entropy of phase $k\left[\mathrm{~J} / \mathrm{m}^{3} \cdot \mathrm{K}\right]$

$S_{k}$ intrinsic specific entropy of phase $k[\mathrm{~J} / \mathrm{kg} . \mathrm{K}]$

Sp Sparrow number, eqn (46) [-]

$S_{s f}^{s}$ specific surface $\left[\mathrm{m}^{2} / \mathrm{m}^{3}\right]$

$t$ time $[\mathrm{s}]$

$T_{D}$ dimensionless temperature, eqn (44) [-]

$T_{k}$ temperature of the phase $k[\mathrm{~K}]$

$\mathbf{u}$ displacement vector of the solid skeleton $[\mathrm{m}]$

$\mathbf{v}_{\text {conv }}$ convective velocity vector $[\mathrm{m} / \mathrm{s}]$

$\mathbf{v}_{k}$ velocity vector of phase $k[\mathrm{~m} / \mathrm{s}]$

$\mathrm{v}^{\infty}$ vertical steady state fluid velocity $[\mathrm{m} / \mathrm{s}]$

$\mathbf{W}$ weighting function [-]

$W$ weighting coefficient used in Remark $5.2[-]$

$X_{F}$ horizontal length of reservoir and formation $[\mathrm{m}]$

$X_{R}$ horizontal length of the reservoir $[\mathrm{m}]$

$X_{W}$ length of the well $[\mathrm{m}]$

$Z_{R}$ height of the reservoir [m]

Greek letters

$\alpha_{T}$ coefficient of thermal conductivity $\left[\mathrm{m}^{2} / \mathrm{s}\right]$

$\Delta(\cdot)$ variation from initial state

$\Delta t$ time step $[\mathrm{s}]$

$\boldsymbol{\epsilon}$ total strain [-]

$\eta_{D}$ dimensionless LTNE parameter, eqn (45) [-]

$\kappa_{s f}$ specific inter-phase heat transfer coeff. $\left[\mathrm{W} / \mathrm{m}^{3} . \mathrm{K}\right]$

$\lambda_{\mathrm{DS}}$ first Lamé's drained modulus [Pa] 
$\Lambda_{k}$ intrinsic thermal conductivity of phase $k$ [W/m.K]

$\mu_{\mathrm{DS}}$ second Lamé's modulus [Pa]

$\mu_{f}$ fracture dynamic viscosity [Pa.s]

$\nu$ drained Poisson's ratio [-]

$\nu_{u}$ undrained Poisson's ratio [-]

$\rho_{k}\left(\rho^{k}\right)$ intrinsic (apparent) density of phase $k\left[\mathrm{~kg} / \mathrm{m}^{3}\right]$

$\boldsymbol{\sigma}$ total stress $[\mathrm{Pa}]$

$\overline{\boldsymbol{\sigma}}$ effective stress $[\mathrm{Pa}]$

$\tau_{\text {SUPG }}$ stabilization parameter $[\mathrm{s}]$

Superscripts and Subscripts

$e$ related to finite element $e$

el elastic contribution

$f$ fracture fluid phase

$s$ solid phase

$s f$ solid-fluid system

$p(v)$ related to pressure (volume)

$T$ thermal

$\mathrm{T}$ transpose

inj injection

out outlet

0 initial

* requires a SUPG stabilization

Operators

|a| absolute value of the scalar a

$|\mathbf{a}|$ Euclidean norm of the vector, tensor a

$\nabla a$ gradient of the scalar $a$

div a divergence of the vector, tensor a

\section{Acknowledgments}

This work is supported by a PhD fellowship from the French Ministry of Higher Education. RG would like to thank the Association Française des Femmes Diplomées des Universités (AFFDU) and the Institut National Polytechnique de Grenoble for their travel grants.

\section{Appendix A. Definition of the vectors $\mathbb{F}^{\text {grav }}$ and $\mathbb{F}^{\text {surf }}$}

$\mathbb{F}^{\text {grav }}$ comprises the gravity contributions to the weak form of the problem (15),

$$
\mathbb{F}^{\text {grav }}=\left[\begin{array}{c}
\int_{V} \mathbf{N}_{\mathbf{u}}^{\mathrm{T}} \rho \mathbf{g} \mathrm{d} V \\
-\int_{V}\left(\nabla \mathbf{N}_{p}\right)^{\mathrm{T}} \frac{k_{f}}{\mu_{f}} \rho_{f} \mathbf{g} \mathrm{d} V \\
0 \\
0 \\
0
\end{array}\right],
$$

while the surface contributions are gathered in 


$$
\mathbb{F}^{\text {surf }}=\left[\begin{array}{c}
\int_{\partial V} \mathbf{N}_{\mathbf{u}}^{\mathrm{T}} \boldsymbol{\sigma} \cdot \hat{\mathbf{n}} \mathrm{d} S \\
\int_{\partial V} \mathbf{N}_{p}^{\mathrm{T}} \mathbf{j}_{f} \cdot \hat{\mathbf{n}} \mathrm{d} S \\
\int_{\partial V} \mathbf{N}_{T}^{\mathrm{T}} \mathbf{q}_{s} \cdot \hat{\mathbf{n}} \mathrm{d} S \\
\int_{\partial V} \mathbf{N}_{T}^{\mathrm{T}} \mathbf{q}_{f} \cdot \hat{\mathbf{n}} \mathrm{d} S
\end{array}\right] .
$$

\section{Appendix B. Definition of the matrices $\mathbb{K}^{e}$ and $\mathbb{D}^{e}$ in eqn (28)}

The submatrices of the element generalized stiffness and diffusion matrices of the finite element formulation (28) are built from constitutive matrices, namely for the generalized stiffness-convection matrix,

$$
\begin{array}{lll}
\mathbf{K}_{\mathbf{u u}}^{e}=\mathbf{E}_{\mathbf{u u}}^{e}, & \mathbf{K}_{\mathbf{u} p_{f}}^{e}=-\xi_{f} \mathbf{C}_{\mathbf{u} p}^{e}, \\
\mathbf{K}_{\mathbf{u} T_{s}}^{e}=-c_{T} / c_{\mathrm{DS}} \mathbf{C}_{\mathbf{u} T}^{e}, & \mathbf{K}_{p_{f} p_{f}}^{e}=-k_{f} / \mu_{f} \mathbf{Q}_{p}^{e}, \\
\mathbf{K}_{T_{s} T_{s}}^{e}=-n_{s} \Lambda_{s} \mathbf{Q}_{T}^{e}-\kappa_{s f} \mathbf{M}_{T T}^{e}, & \mathbf{K}_{T_{s} T_{f}}^{e}=\kappa_{s f} \mathbf{M}_{T T}^{e} .
\end{array}
$$

and for the generalized diffusion matrix,

$$
\begin{array}{ll}
\mathbf{D}_{p_{f} \mathbf{u}}^{e}=-\xi_{f}\left(\mathbf{C}_{\mathbf{u} p}^{e}\right)^{\mathrm{T}}, & \mathbf{D}_{p_{f} p_{f}}^{e}=-a_{f f} \mathbf{M}_{p p}^{e}, \\
\mathbf{D}_{p_{f} T_{s}}^{e}=-a_{f T_{s}} \mathbf{M}_{p T}^{e}, & \mathbf{D}_{p_{f} T_{f}}^{e}=-a_{f T_{f}} \mathbf{M}_{p T}^{e}, \\
\mathbf{D}_{T_{s} \mathbf{u}}^{e}=-T_{s} c_{T} / c_{\mathrm{DS}}\left(\mathbf{C}_{\mathbf{u} T}^{e}\right)^{\mathrm{T}}, & \mathbf{D}_{T_{s} p_{f}}^{e}=-T_{s} a_{f T_{s}}\left(\mathbf{M}_{p T}^{e}\right)^{\mathrm{T}}, \\
\mathbf{D}_{T_{s} T_{s}}^{e}=-a_{T_{s} T_{s}} \mathbf{M}_{T T}^{e} . &
\end{array}
$$

Some sub-matrices of the element matrices $\mathbb{K}^{e}$ and $\mathbb{D}^{e}$ are discretized with the SUPG method namely,

$$
\begin{array}{lll}
{ }^{*} \mathbf{K}_{T_{f} p_{f}}^{e}=-n_{f}\left(1-T_{f} c_{f T}\right){ }^{*} \mathbf{C}_{T p}^{e}, & { }^{*} \mathbf{K}_{T_{f} T_{s}}^{e}=\kappa_{s f}{ }^{*} \mathbf{M}_{T T}^{e}, \\
{ }^{*} \mathbf{K}_{T_{f} T_{f}}^{e}=-n_{f} \Lambda_{f} \mathbf{Q}_{T}^{e}-\kappa_{s f}{ }^{*} \mathbf{M}_{T T}^{e} & -n_{f} \rho_{f} C_{f}^{(p)}{ }^{*} \mathbf{C}_{T T}^{e}, \\
{ }^{*} \mathbf{D}_{T_{f} p_{f}}^{e}=-T_{f} a_{f T_{f}}{ }^{*} \mathbf{M}_{T p}^{e}, & { }^{*} \mathbf{D}_{T_{f} T_{f}}^{e}=-a_{T_{f} T_{f}}{ }^{*} \mathbf{M}_{T T}^{e} .
\end{array}
$$

The material properties have been aggregated in the coefficients,

$$
\begin{array}{lll}
a_{f f} & =n_{f} c_{f H}+\left(\xi_{f}-n_{f}\right) c_{s}, & a_{f T_{s}}=-\left(\xi_{f}-n_{f}\right) c_{T}, \\
a_{f T_{f}} & =-n_{f} c_{f T}, & a_{T_{s} T_{s}}=n_{s} \rho_{s} C_{s}^{(v)}, \\
a_{T_{f} T_{f}} & =n_{f} \rho_{f} C_{f}^{(p)} &
\end{array}
$$

The finite element sub-matrices of the compact weak formulation in equations (B.1) to (B.3) are now provided in explicit form. First, the three matrices involved in the elementary contribution to the balance of momentum of the mixture,

$$
\begin{aligned}
& \mathbf{E}_{\mathbf{u u}}^{e}=\int_{V^{e}}\left(\mathbf{B}_{\mathbf{u}}\right)^{\mathrm{T}} \mathbf{D}_{e l} \mathbf{B}_{\mathbf{u}} d V^{e}, \quad \mathbf{C}_{\mathbf{u} p}^{e}=\int_{V^{e}}\left(\nabla \mathbf{N}_{\mathbf{u}}\right)^{\mathrm{T}} \mathbf{N}_{p} d V^{e}, \\
& \mathbf{C}_{\mathbf{u} T}^{e}=\int_{V^{e}}\left(\nabla \mathbf{N}_{\mathbf{u}}\right)^{\mathrm{T}} \mathbf{N}_{T} d V^{e},
\end{aligned}
$$


where $\mathbf{D}_{e l}$ is the drained stiffness matrix and $\mathbf{B}_{\mathbf{u}}$ is the strain displacement matrix. Next, the matrices involved in the diffusion phenomena,

$$
\mathbf{Q}_{p}^{e}=\int_{V^{e}}\left(\nabla \mathbf{N}_{p}\right)^{\mathrm{T}} \nabla \mathbf{N}_{p} d V^{e}, \quad \mathbf{Q}_{T}^{e}=\int_{V^{e}}\left(\nabla \mathbf{N}_{T}\right)^{\mathrm{T}} \nabla \mathbf{N}_{T} d V^{e} .
$$

The mass matrices below are discretized with the Galerkin method,

$$
\begin{aligned}
& \mathbf{M}_{p p}^{e}=\int_{V^{e}}\left(\mathbf{N}_{p}\right)^{\mathrm{T}} \mathbf{N}_{p} d V^{e}, \quad \mathbf{M}_{T T}^{e}=\int_{V^{e}}\left(\mathbf{N}_{T}\right)^{\mathrm{T}} \mathbf{N}_{T} d V^{e}, \\
& \mathbf{M}_{p T}^{e}=\int_{V^{e}}\left(\mathbf{N}_{p}\right)^{\mathrm{T}} \mathbf{N}_{T} d V^{e}
\end{aligned}
$$

while the following matrices are discretized with the SUPG method,

$$
{ }^{*} \mathbf{M}_{T T}^{e}=\int_{V^{e}}\left(\mathbf{W}_{T}\right)^{\mathrm{T}} \mathbf{N}_{T} d V^{e}, \quad{ }^{*} \mathbf{M}_{T p}^{e}=\int_{V^{e}}\left(\mathbf{W}_{T}\right)^{\mathrm{T}} \mathbf{N}_{p} d V^{e} .
$$

Two convective matrices associated with the element contributions of the balance of energy of the fluid phase are required:

$$
{ }^{*} \mathbf{C}_{T T}^{e}=\int_{V^{e}}\left(\mathbf{W}_{T}\right)^{\mathrm{T}} \mathbf{v}_{\mathrm{conv}} \cdot \nabla \mathbf{N}_{T} d V^{e}, \quad{ }^{*} \mathbf{C}_{T p}^{e}=\int_{V^{e}}\left(\mathbf{W}_{T}\right)^{\mathrm{T}} \mathbf{v}_{\mathrm{conv}} \cdot \nabla \mathbf{N}_{p} d V^{e}
$$

The $[1 \times 4]$ vectors of shape functions,

$$
\mathbf{N}_{p}=\mathbf{N}_{T}=\left[N^{1} N^{2} N^{3} N^{4}\right]
$$

are identical for the pressure and temperature fields. $N^{1}, N^{2}, N^{3}$ and $N^{4}$ are the shape functions of the Q4 elements. $\mathbf{N}_{\mathbf{u}}$ is the expanded shape function vector of size $[2 \times 8]$.

\section{Appendix C. An expression for the specific surface}

The empirical expression of the specific surface described in the literature applies to fluid flow through packed beds. The starting point is the specific area of a solid sphere bathed in fluid at maximum density in a cube of side length $d_{p}$,

$$
S_{s f}^{s}=\frac{\text { solid area }}{\text { total volume }}=\frac{\pi d_{p}^{2}}{d_{p}^{3}} .
$$

In this situation, the solid porosity $n_{s}$ is equal to $\pi / 6$. Hence, by assuming that the wetted surface is equal to the solid-fluid specific surface, the specific surface is related to the solid porosity $n_{s}$ and to the solid characteristic dimension $d_{p}$,

$$
S_{s f}^{s}=\frac{6 n_{s}}{d_{p}} .
$$

Although satisfactory for small particles $[38,49,50]$ eqn (C.2) is not adapted for the solid-fluid specific surface area of HDR reservoirs endowed with rock blocks with a spacing larger than aperture $B \gg 2 b$. To the best knowledge of the authors, no experimental result is available to evaluate $S_{s f}^{s}$ for HDR reservoirs. A theoretical formula $a d-h o c$ to our 2D plane strain problem is proposed. The specific surface $S_{s f}^{s}$ is obtained by considering four fluid rectangles of size $b \times(B+b)$ surrounding a solid square of area $B^{2}$ and of wetted length $4 \times B$ in a surface $S=(B+2 b)^{2}$,

$$
S_{s f}^{s}=\frac{\text { fluid length }}{\text { total surface }}=\frac{4 \times B}{S} .
$$

The total surface may be substituted by the fluid porosity $n_{f}=4 b(B+b) / S$, leading to (40) for $2 b \ll B$. This relation highlights the importance, at constant aperture $2 b$, of the fluid porosity $n_{f}$ in the heat transfer mechanism. Still, note that its validity has not been tested experimentally. 

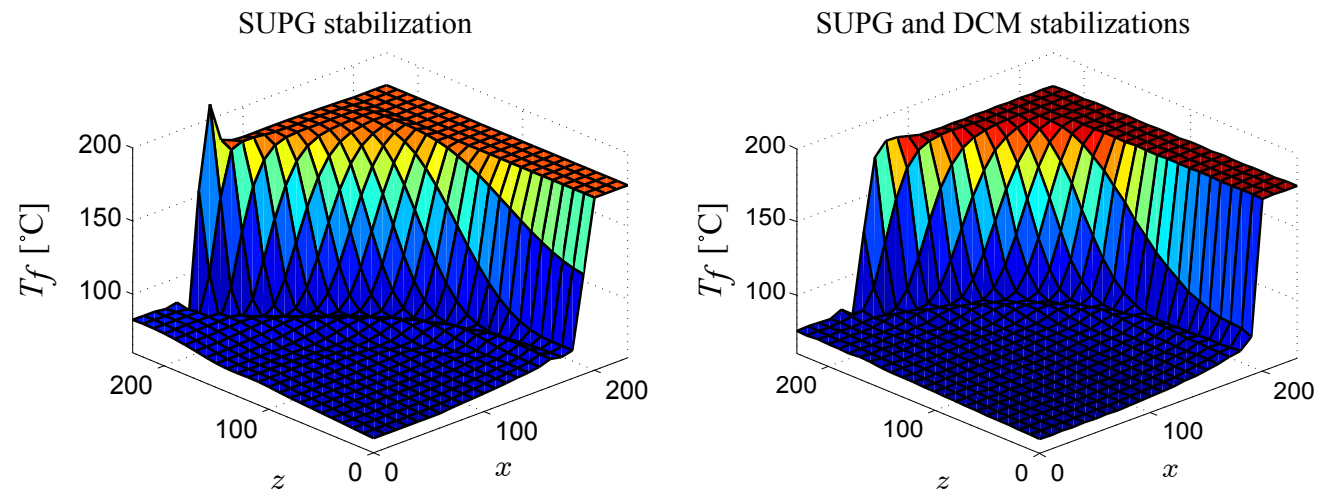

Figure D.15: Contours of fluid temperature for a steady state fluid velocity $\mathrm{v}^{\infty}=9.0 \times 10^{-3} \mathrm{~m} / \mathrm{s}$ at time $t=0.38$ hour, accounting for heat transfer with the surrounding, with the SUPG stabilization (left) and with the SUPG and DC stabilizations (right). The DC stabilization effectively cures some overshootings that are not taken care of by the SUPG method.

\section{Appendix D. The discontinuity capturing method}

The shock capturing operator proposed by Tezduyar and Park [51] aims at improving the SUPG stabilization to smoothly resolve sharp layers. The discontinuity capturing (DC) stabilization suggests, in place of (16), the weighting function of the form,

$$
\mathbf{W}_{T}=\mathbf{N}_{T}+\tau_{\mathrm{SUPG}} \mathbf{v}_{\text {conv }} \cdot \nabla \mathbf{N}_{T}+\tau_{\mathrm{DC}} \mathbf{v}_{\text {conv } \|} \cdot \nabla \mathbf{N}_{T},
$$

in which $\mathbf{v}_{\text {conv } \|}$ is the projection of $\mathbf{v}_{\text {conv }}$ on the direction of the gradient $\mathbf{i}=\nabla T_{f} /\left|\nabla T_{f}\right|$ if $\nabla T_{f} \neq \mathbf{0}$, namely,

$$
\mathbf{v}_{\text {conv } \|}=\left(\mathbf{v}_{\text {conv }} \cdot \mathbf{i}\right) \mathbf{i},
$$

and $\nabla T_{f}$ is obtained consistently with the element discretization $T_{f}=\mathbf{N}_{T} T_{f}^{e}$. If $\nabla T_{f}$ vanishes, then so does $\mathbf{v}_{\text {conv } \|}$. This method is seen to be non-linear since the projection depends on the solution.

Tezduyar and Park [51] proposed to define the stabilization parameter $\tau_{\mathrm{DC}}$ as a function of the direction $\nabla T_{f}$ with respect to the flow and of its magnitude as,

$$
\tau_{\mathrm{DC}}=\frac{h_{\|}}{2\left|\mathbf{v}_{\mathrm{conv} \|}\right|} h_{\|} \frac{\left|\nabla T_{f}\right|}{T_{\mathrm{ref}, f}} \eta(p),
$$

where $T_{\mathrm{ref}, f}$ is a reference value of the unknown $T_{f}$ and $h_{\|}$is the element size in the direction of the thermal gradient,

$$
h_{\|}=\frac{2\left|\mathbf{v}_{\mathrm{conv} \|}\right|}{\sum_{a}\left|\mathbf{v}_{\mathrm{conv} \|} \cdot \nabla N_{T}^{a}\right|} .
$$

The argument $p=\left|\mathbf{v}_{\text {conv } \|}\right| /\left|\mathbf{v}_{\text {conv }}\right|$ of the function $\eta$ remains in the interval $[0,1]$, and the function $\eta(p)$ is designed to vanish at the end of its domain, that is, whenever the velocity $\mathbf{v}_{\text {conv }}$ and the gradient $\nabla T_{f}$ are either perpendicular $(p=0)$ or parallel $(p=1)$. Tezduyar and Park [51] take $\eta(p)=2 p(1-p)$. The DC method acts only on thermal gradient oblique to the flow. For a thermal gradient orthogonal to the flow, both the velocity $\mathbf{v}_{\text {conv } \|}$ and $\eta$ vanish, and for a thermal gradient parallel to the flow, $\eta=0$ so that the SUPG stabilization is not doubled.

The discontinuity capturing method is illustrated by Fig. D.15. While it damps some overshootings which are not cured by the SUPG stabilization, the DC stabilization has also drawbacks: 1 . the number of iterations per time step is heavily increased, and 2. the fully coupled thermo-hydro-mechanical model fails to converge at large times and large pumping rates.

[1] H. Tenzer, 2001. Development of hot dry rock technology, Bulletin Geo-Heat Center, 32, 14-22. 
[2] H.C.H. Armstead and J.W. Tester, 1987. Heat mining, E.\&F.N. Spon Ltd., London and New York.

[3] K. Evans, B. Valley, M. Häring, R.J. Hopkirk, C. Baujard, T. Kohl, T. Mégel, L. André, S. Portier and F.D. Vuataz, 2009. Studies and support for the EGS reservoirs at Soultz-sous-Forêts, Final report April 2004 - May 2009.

[4] A.C. Gringarten, P.A. Witherspoon and Y. Ohnishi, 1975. Theory of heat extraction from fractured hot dry rock, Journal of Geophysical Research, 80, 1120-1124.

[5] D. Elsworth, 1989. Theory of Thermal Recovery From a Spherically Stimulated Hot Dry Rock Reservoir, Journal of Geophysical Research, 94, 1927-1934.

[6] A.H.D. Cheng, A. Ghassemi and E. Detournay, 2001. Integral equation solution of heat extraction from a fracture in hot dry rock, International Journal for Numerical and Analytical Methods in Geomechanics, 25(13), 1327-1338.

[7] A. Ghassemi, S. Tarasovs and A.H.D. Cheng, 2005. Integral equation solution of heat extraction-induced thermal stress in enhanced geothermal reservoirs, International Journal for Numerical and Analytical Methods in Geomechanics, 29, $829-844$.

[8] Y. Wang and M.B. Dusseault, 2003. A coupled conductive-convective thermo-poroelastic solution and implications for wellbore stability, Journal of Petroleum Science, 38, 187-198.

[9] K. Hayashi, J. Willis-Richards, R.J. Hopkirk and Y. Niibori, 1999. Numerical models of HDR geothermal reservoirs - a review of current thinking and progress, Geothermics, 28, 507-518.

[10] M.J. O'Sullivan, K. Pruess and M.J. Lippmann, 2001. State of the art of geothermal reservoir simulation, Geothermics, 30(4), 395-429.

[11] R. DuTeaux, D. Swenson and B. Hardeman, 1996. Insight from modelling discrete fractures using GEOCRACK, Proceedings, Twenty-First Workshop on Geothermal Reservoir Engineering Stanford University, Stanford. California, January 22-24, 287-293.

[12] D. Bruel, 2002. Impact of Induced Thermal Stresses During Circulation Tests in an Engineered Fractured Geothermal Reservoir: Example of the Soultz-Sous-Forêts European Hot Fractured Rock Geothermal Project, Rhine Graben, France, Oil \& Gas Science and Technology, 57, 459-470.

[13] A. Bataillé, P. Genthon, M. Rabinowicz and B. Fritz, 2006. Modeling the coupling between free and forced convection in a vertical permeable slot: implications for the heat production of an Enhanced Geothermal System, Geothermics, 35, 654-682.

[14] T. Kohl and R.J. Hopkirk, 1995. "FRACure" - A simulation code for forced fluid flow and transport in fractured, porous rock, Geothermics, 24, 333-343

[15] K.M. Bower and G. Zyvoloski, 1997. A numerical model for thermo-hydro-mechanical coupling in fractured rock, International Journal of Rock Mechanics and Mining Sciences, 34, 1201-1211.

[16] J. Taron, D. Elsworth and K-B. Min, 2009. Numerical simulation of thermal-hydrologic-mechanical-chemical processes in deformable, fractured porous media, International Journal of Rock Mechanics \& Mining Sciences, 46, 842-854.

[17] B. Loret and N. Khalili, 2000. A three-phase model for unsaturated soils, International Journal for Numerical and Analytical Methods in Geomechanics, 24, 893-927.

[18] N. Khalili and B. Loret, 2001. An Elasto-plastic Model for Non-Isothermal Analysis of Flow and Deformation in Unsaturated Porous Media Formulation, International Journal of Solids and Structures, 38, 8305-8330.

[19] J. Taron and D. Elsworth, 2009. Thermal-hydrologic-mechanical-chemical processes in the evolution of engineered geothermal reservoirs, International Journal of Rock Mechanics \& Mining Sciences, 46, 855-864.

[20] E. C. Aifantis, 1980. Further comments on the problem of heat extraction from hot dry rocks, Mechanics of Research Communications, 7(4), 219-226.

[21] J. Willis-Richards and T. Wallroth, 1995. Approaches to the modelling of HDR reservoirs: a review, Geothermics, 24, 307-332.

[22] L. Virto, M. Carbonell, R. Castilla and P.J. Gamez-Montero, 2009. Heating of saturated porous media in practice: several causes of local thermal non-equilibrium, International Journal of Heat and Mass Transfer, 52, 5412-5422.

[23] A.C. Eringen and J.D. Ingram, 1965. A continuum theory of chemically reacting media, International Journal of Engineering Science, 3, 197-212.

[24] R.M. Bowen and P.J. Chen, 1975. Waves in a binary mixture of linear elastic materials, Journal de Mécanique, 14, $237-266$.

[25] B. Loret, 2008. Biomechanical aspects of soft tissues, unpublished lecture notes.

[26] R. Gelet, 2011. Thermo-hydro-mechanical study of deformable porous media with double porosity in local thermal nonequilibrium, PhD thesis, Institut National Polytechnique de Grenoble, France, and The University of New South Wales, Sydney, Australia.

[27] A.N. Brooks and T.J.R. Hughes, 1982. Streamline upwind/Petrov-Galerkin formulations for convection dominated flows with particular emphasis on the incompressible Navier-Stokes equations, Computer Methods in Applied Mechanics and Engineering, 32, 199-259.

[28] T.P. Fries and H.G. Matthies, 2004. A Review of Petrov-Galerkin Stabilization Approaches and an Extension to Meshfree Methods, Informatikbericht Nr. 2004-01, Technical University Braunschweig, Brunswick, Germany.

[29] T.E. Tezduyar and Y. Osawa, 2000. Finite element stabilization parameters computed from element matrices and vectors, Computer Methods in Applied Mechanics and Engineering, 190, 411-430.

[30] T. Belytschko and T.J.R. Hughes, 1983. Computational methods for transient analysis, North-Holland, Computational Methods in Mechanics, Amsterdam.

[31] A. Bejan, 1993. Heat Transfer, John Wiley \& Sons, New York, 698 p.

[32] J.B. Warren and P.J. Root, 1963. The Behaviour of Naturally Fractured Reservoirs, Society of Petroleum Engineers Journal, 228, 245-255. 
[33] N. Wakao and S. Kaguei, 1982. Heat and Mass Transfer in Packed Beds, Gordon and Breach, Science Publishers, New York.

[34] F. Zanotti and R.G. Carbonell, 1984. Development of Transport Equations for Multiphase Systems, I-II-III, Chemical Engineering Science, 39, 263-278, 279-297, 299-311.

[35] C. Pecker and H. Deresiewicz, 1973. Thermal effects on wave propagation in liquid-filled porous media, Acta Mechanica, 16, 45-64.

[36] P.X. Jiang, R.N. Xu and W. Gong, 2006. Particle-to-fluid heat transfer coefficients in miniporous media, Chemical Engineering Science, 61, 7213-7222.

[37] W.J. Minkowycz, A. Haji-Sheikh and K. Vafai, 1999. On departure from local thermal equilibrium in porous media due to a rapidly changing heat source: the Sparrow number, International Journal of Heat and Mass Transfer, 42, 3373-3385.

[38] D.A. Nield, A.V. Kuznetsov and M. Xiong, 2002. Effect of local thermal non-equilibrium on thermally developing forced convection in a porous medium, International Journal of Heat and Mass Transfer, 45, 4949-4955.

[39] R. Gelet, B. Loret and N. Khalili, 2012. A thermo-hydro-mechanical model in local thermal non-equilibrium for fractured HDR reservoirs with double porosity, Journal of Geophysical Research, 117, doi:10.1029/2012JB009161.

[40] N. Khalili, S. Valliappan and C.F. Wan, 1999. Consolidation of Fissured Clays, Géotechnique, 49(1), 75-89.

[41] T.J.R. Hughes, M. Mallet and A. Mizukami, 1986. A new finite element formulation for computational fluid dynamics: II. Beyond SUPG, Computer Methods in Applied Mechanics and Engineering, 1986, 54(3), 341-355.

[42] G.A. Zyvoloski, R.L. Aamodt and R.G. Aguilar, 1981. Evaluation of the second hot dry rock geothermal energy reservoir: results of Phase I, Run Segment 5, Los Alamos National Laboratory, NM (USA).

[43] H.D. Murphy, R.G. Lawton, J.W. Tester, R.M. Potter, D.W. Brow and R.L. Aamodt, 1977. Preliminary assessment of a geothermal energy reservoir formed by hydraulic fracturing, Society of Petroleum Engineers Journal, 17, 317-326.

[44] N. Watanabe, W. Wang, C.I. McDermott, T. Taniguchi and O. Kolditz, 2010. Uncertainty analysis of thermo-hydromechanical coupled processes in heterogeneous porous media, Computational Mechanics, 45, 263-280.

[45] G. de Marsily, 1981. Quantitative Hydrogeology - Groundwater Hydrology for Engineers, Academic Press, Masson, Paris.

[46] S.R. Idelsohn, J.C. Heinrich and E. Oñate, 1998. Petrov-Galerkin methods for the transient advective-diffusive equation with sharp gradients, International Journal for Numerical Methods in Engineering, 39, 1455-1473.

[47] S. Yin, B.F. Towler, M.B. Dusseault and L. Rothenburg, 2010. Fully Coupled THMC Modeling of Wellbore Stability with Thermal and Solute Convection Considered, Transport in Porous Media, 84, 773-798.

[48] A. Ghassemi, A. Nygren and A. Cheng, 2008. Effects of heat extraction on fracture aperture: A poro-thermoelastic analysis, Geothermics, 37, 525-539.

[49] B. Alazmi and K. Vafai, 2000. Analysis of variants within the porous media transport models, Journal of Heat Transfer, $122,303-326$.

[50] D.A. Nield and A. Bejan, 2006. Convection in porous media, Springer Verlag.

[51] T.E. Tezduyar and Y.J. Park, 1986. Discontinuity-capturing finite element formulations for nonlinear convection-diffusionreaction equations, Computer Methods in Applied Mechanics and Engineering, 59(3), 307-325. 